\title{
Slow dynamics in critical ferromagnetic vector models relaxing from a magnetized initial state
}

\author{
Pasquale Calabrese ${ }^{1,2}$ and Andrea Gambassi ${ }^{3,4}$ \\ ${ }^{1}$ Institute for Theoretical Physics, Amsterdam University, Valckenierstraat 65, 1018 XE \\ Amsterdam, The Netherlands. \\ ${ }^{2}$ Dipartimento di Fisica dell'Università di Pisa and INFN, Pisa, Italy. \\ ${ }^{3}$ Max-Planck-Institut für Metallforschung, Heisenbergstr. 3, D-70569 Stuttgart, Germany. \\ ${ }^{4}$ Institut für Theoretische und Angewandte Physik, Universität Stuttgart, Pfaffenwaldring 57, \\ D-70569 Stuttgart, Germany.
}

(October 10, 2006)

\begin{abstract}
Within the universality class of ferromagnetic vector models with $O(n)$ symmetry and purely dissipative dynamics, we study the non-equilibrium critical relaxation from a magnetized initial state. Transverse correlation and response functions are exactly computed for Gaussian fluctuations and in the limit of infinite number $n$ of components of the order parameter. We find that the fluctuation-dissipation ratios (FDRs) for longitudinal and transverse modes differ already at the Gaussian level. In these two exactly solvable cases we completely describe the crossover from the short-time to the long-time behavior, corresponding to a disordered and a magnetized initial condition, respectively. The effects of non-Gaussian fluctuations on longitudinal and transverse quantities are calculated in the first order in the $\epsilon$-expansion $(\epsilon=$ $4-d)$ and reliable three-dimensional estimates of the two FDRs are obtained.
\end{abstract}




\section{INTRODUCTION}

Consider a ferromagnetic system with vector order parameter $\Phi_{i}(\mathbf{x}, t), i=1, \ldots, n$, a critical point at temperature $T_{c}$ and at vanishing external magnetic field $\mathbf{h}_{c}=0$, and prepare it in some initial configuration (which might correspond to an equilibrium state at a given temperature $T_{0}$ and magnetic field $\mathbf{h}_{0}$ ). At time $t=0$ bring the system in contact with a thermal bath of temperature $T_{b} \neq T_{0}$ in the presence of a magnetic field $\mathbf{h}_{b}$. The ensuing relaxation process is characterized by some equilibration time $t_{\text {eq }}$ so that for $t \gg t_{\text {eq }}$ equilibrium is attained, whereas for $0<t \ll t_{\text {eq }}$, the evolution depends on the specific initial condition. Upon approaching the critical point $T_{b}=T_{c}, \mathbf{h}_{b}=\mathbf{h}_{c}=0$ the equilibration time diverges and therefore equilibrium is never achieved. One-time quantities (like meanenergy, magnetization, etc.) typically display a slow relaxation (power-law) towards their equilibrium values, even in the extreme situation in which $t_{\text {eq }}$ is infinite. Consequently, the natural objects to monitor the non-equilibrium evolution of the system are two-time quantities, like the time-dependent correlation function of some local observable $\mathcal{O}$ which is given by $C_{\mathbf{x}}^{\mathcal{O}}(t, s)=\langle\mathcal{O}(\mathbf{x}, t) \mathcal{O}(\mathbf{0}, s)\rangle$, $(\langle\ldots\rangle$ stands for the average over the stochastic dynamics) and the linear response $R_{\mathbf{x}}^{\mathcal{O}}(t, s)$ to an external field $h_{\mathcal{O}}$ conjugate to $\mathcal{O}$. $R_{\mathbf{x}}^{\mathcal{O}}(t, s)$ is defined by $R_{\mathbf{x}}^{\mathcal{O}}(t, s)=\delta\langle\mathcal{O}(\mathbf{x}, t)\rangle /\left.\delta h_{\mathcal{O}}(s)\right|_{h_{\mathcal{O}}=0}$, where $h_{\mathcal{O}}$ is applied at time $s>0$ at the point $\mathbf{x}=0$. For $t>s \gg t_{\text {eq }}$ correlation and response functions take their equilibrium forms - depending only on $t-s$ - and they are related by the fluctuation-dissipation theorem (FDT)

$$
T_{b} R_{\mathbf{x}}^{\mathcal{O}}\left(t \gg t_{\mathrm{eq}}, s \gg t_{\mathrm{eq}}\right)=\partial_{s} C_{\mathbf{x}}^{\mathcal{O}}\left(t \gg t_{\mathrm{eq}}, s \gg t_{\mathrm{eq}}\right) .
$$

This is no longer true in the so-called aging regime: correlation and response functions have a non-trivial (homogeneous) dependence on both $t$ and $s$, even for very large times. In particular their decays as functions of $t$ become slower upon increasing $s$, the age of the system. This is why this evolution is referred to as aging.

The FDT suggests the introduction of the so-called fluctuation-dissipation ratio (FDR) $[1,2]$ :

$$
X_{\mathbf{x}}^{\mathcal{O}}(t, s)=\frac{T_{b} R_{\mathbf{x}}^{\mathcal{O}}(t, s)}{\partial_{s} C_{\mathbf{x}}^{\mathcal{O}}(t, s)}
$$

which can be used in order to understand the exten to which a system is evolving out of equilibrium. The asymptotic value of the FDR

$$
X_{\mathcal{O}}^{\infty}=\lim _{s \rightarrow \infty} \lim _{t \rightarrow \infty} X_{\mathbf{x}=0}^{\mathcal{O}}(t, s)
$$

is a useful quantity in the description of systems with slow dynamics, since $X_{\mathcal{O}}^{\infty}=1$ whenever the aging evolution is interrupted and the system crosses over (for $t>s \gg t_{\text {eq }}$ ) to equilibrium dynamics. Conversely, $X_{\mathcal{O}}^{\infty} \neq 1$ is a signal of an asymptotic non-equilibrium dynamics.

Within the field-theoretical approach to critical dynamics it is more convenient to focus on the behavior of observables in momentum space. Accordingly, hereafter we mainly consider the momentum-dependent response $R_{\mathbf{q}}^{\mathcal{O}}(t, s)$ and correlation $C_{\mathbf{q}}^{\mathcal{O}}(t, s)$ functions, defined as the Fourier transforms of $R_{\mathbf{x}}^{\mathcal{O}}(t, s)$ and $C_{\mathbf{x}}^{\mathcal{O}}(t, s)$, respectively. In momentum space it is natural to introduce a quantity with the same role as $X_{\mathbf{x}}^{\mathcal{O}}(t, s)[3]$ :

$$
\mathcal{X}_{\mathbf{q}}^{\mathcal{O}}(t, s)=\frac{T_{b} R_{\mathbf{q}}^{\mathcal{O}}(t, s)}{\partial_{s} C_{\mathbf{q}}^{\mathcal{O}}(t, s)} .
$$


It has been argued that the long-time limit

$$
\mathcal{X}_{\mathcal{O}}^{\infty} \equiv \lim _{s \rightarrow \infty} \lim _{t \rightarrow \infty} \mathcal{X}_{\mathbf{q}=0}^{\mathcal{O}}(t, s)
$$

has exactly the same value as $X_{\mathcal{O}}^{\infty}[3]$ (see also Ref. [4]).

Aging was known to occur in disordered and complex systems (see, e.g., Ref. [5]) and only recently attention has been focused on simpler critical systems such as ferromagnets, whose universal behaviors can be rather easily studied by using powerful theoretical methods and which might provide insight into more general cases [6] (see Ref. [7] for a pedagogical introduction). In particular it was argued that $X^{\infty}$ is a universal amplitude ratio for quenches to $T_{c}[8]$. In this respect several dynamic universality classes were investigated and the scaling forms the of response and correlation functions, together with the associated FDRs were calculated by means of exact, approximate, or numerical methods [9-30] (see Ref. [6] for a review).

In this paper we consider the purely dissipative dynamics (Model A, according to the classification of Ref. [31]) of the $O(n)$ symmetric model relaxing from a magnetized state (in a sense that will be specified below). Previous works [32,33,30] mainly focused on the case $n=1$ - corresponding to the Ising universality class - because the dynamics of actual systems with vector order parameter and $O(n>1)$ symmetry typically possesses conservation laws which are not accounted for by Model A. For example, an isotropic ferromagnet (antiferromagnet) is correctly described by the so-called Model J (G) universality class [31]. Nonetheless, we consider this unphysical model because it is probably the simplest example in which the dynamics is affected by two different fluctuation modes. In fact, consider the relaxation from an initial state with a non-vanishing magnetization $\mathbf{M}_{0}$. In the absence of external fields the magnetization will evolve as $M(t)$ in the direction $\widehat{\mathbf{M}}_{0} \equiv \mathbf{M}_{0} / M_{0}$. The time evolution of the component of the order parameter parallel to $\mathbf{M}(t)$ is clearly different from the evolution of the $n-1$ components perpendicular to it, which are characterized by a residual $O(n-1)$ symmetry. In what follows the mode $\sigma(\mathbf{x}, t)=\Phi(\mathbf{x}, t) \cdot \widehat{\mathbf{M}}_{0}-M(t)$ parallel to $\widehat{\mathbf{M}}_{0}$ is referred to as longitudinal, whereas the $n-1$ perpendicular modes $\boldsymbol{\pi}(\mathbf{x}, t)=\Phi(\mathbf{x}, t)-\widehat{\mathbf{M}}_{0}\left[\widehat{\mathbf{M}}_{0} \cdot \Phi(\mathbf{x}, t)\right]$ are referred to as transverse. They are characterized by different responses and correlations, with FDRs:

$$
X_{\sigma}^{\infty}=\lim _{s \rightarrow \infty} \lim _{t \rightarrow \infty} \frac{T_{b} R_{\mathbf{q}=0}^{\sigma}(t, s)}{\partial_{s} C_{\mathbf{q}=0}^{\sigma}(t, s)}, \quad X_{\boldsymbol{\pi}}^{\infty}=\lim _{s \rightarrow \infty} \lim _{t \rightarrow \infty} \frac{T_{b} R_{\mathbf{q}=0}^{\boldsymbol{\pi}}(t, s)}{\partial_{s} C_{\mathbf{q}=0}^{\boldsymbol{\pi}}(t, s)} .
$$

In spite of its unphysical nature, the purely dissipative dynamics of the $O(n)$ model has a rich behavior which is easily accessible to numerical simulations and possibly captures some of the features of more realistic dynamics. The model has already been studied by Fedorenko and Trimper [24] in a dimensional expansion close to the lower critical dimension - an approach that is complementary to the one adopted here.

The paper is organized as follows. In Sec. II we introduce the model and we discuss the general scaling forms for the correlation and response functions of longitudinal and transverse modes after a quench from a magnetized state. In Sec. III we solve the model within the Gaussian approximation. In Sec. IV we derive the exact transverse response and correlation functions in the limit of infinite number of components of the order parameter. 
In these two exactly solvable cases we describe completely the crossover from the short-time to the long-time behavior, corresponding to a disordered and a magnetized initial condition, respectively. In Sec. V we account for non-Gaussian fluctuations up to first-order in the $\epsilon$-expansion $(\epsilon=4-d)$ and from the resulting expressions of the response and correlation functions we calculate the associated FDR. Finally in Sec. VI we summarize and discuss our results.

\section{THE MODEL}

The purely dissipative dynamics of a $n$-component field $\Phi=\left(\Phi_{1}, \ldots, \Phi_{n}\right)$ can be specified in terms of the stochastic Langevin equation

$$
\partial_{t} \Phi_{i}(\mathbf{x}, t)=-\Omega \frac{\delta \mathcal{H}[\Phi]}{\delta \Phi_{i}(\mathbf{x}, t)}+\xi_{i}(\mathbf{x}, t)
$$

where $\Omega$ is the kinetic coefficient, $\xi_{i}(\mathbf{x}, t)$ a set of zero-mean stochastic Gaussian noises with

$$
\left\langle\xi_{i}(\mathbf{x}, t) \xi_{j}\left(\mathbf{x}^{\prime}, t^{\prime}\right)\right\rangle=2 \delta_{i j} \Omega \delta\left(\mathbf{x}-\mathbf{x}^{\prime}\right) \delta\left(t-t^{\prime}\right)
$$

and $\mathcal{H}[\Phi]$ is the static Hamiltonian. Near the critical point, $\mathcal{H}[\Phi]$ may be assumed of the $O(n)$-symmetric Landau-Ginzburg form

$$
\mathcal{H}[\Phi]=\int \mathrm{d}^{d} x\left[\frac{1}{2}(\nabla \Phi)^{2}+\frac{r_{0}}{2} \Phi^{2}+\frac{g_{0}}{4 !}\left(\Phi^{2}\right)^{2}\right],
$$

where $r_{0}$ is a parameter that has to be tuned to a critical value $r_{0, c}$ in order to approach the critical temperature $T=T_{c}\left(r_{0, c}=0\right.$, within the analytical approach discussed below $)$, and $g_{0}>0$ is the bare coupling constant of the theory.

Correlation and response functions of a field which evolves according to the Langevin equation (7) can be obtained by means of the field-theoretical action [34,35]

$$
S[\Phi, \tilde{\Phi}]=\int_{0}^{\infty} \mathrm{d} t \int \mathrm{d}^{d} x\left[\tilde{\Phi} \partial_{t} \Phi+\Omega \tilde{\Phi} \frac{\delta \mathcal{H}[\Phi]}{\delta \Phi}-\tilde{\Phi} \Omega \tilde{\Phi}\right]
$$

where $\tilde{\Phi}(\mathbf{x}, t)$ is an auxiliary field, conjugate to the external field $\mathbf{h}$ in such a way that $\mathcal{H}[\Phi, \mathbf{h}]=\mathcal{H}[\Phi]-\int \mathrm{d}^{d} x \mathbf{h} \cdot \Phi$.

The effect of a macroscopic initial condition $\Phi_{0}(\mathbf{x}) \equiv \Phi(\mathbf{x}, t=0)$ may be accounted for by averaging over the initial configuration with a weight $\mathrm{e}^{-H_{0}\left[\Phi_{0}\right]}$, where $[36,37]$

$$
H_{0}\left[\Phi_{0}\right]=\int \mathrm{d}^{d} x \frac{\tau_{0}}{2}\left[\Phi_{0}(\mathbf{x})-\mathbf{M}_{0}\right]^{2}
$$

specifies an initial state with Gaussian distribution of the order parameter fluctuations, short-range correlations proportional to $\tau_{0}^{-1}$, and spatially constant average order parameter $\mathbf{M}_{0}=\left\langle\Phi_{0}(\mathbf{x})\right\rangle$. In the experimental protocols we have in mind the initial state with $\mathbf{M}_{0} \neq 0$ can be obtained by equilibrating the system for $t<0$ in the presence of an external field $\mathbf{h}_{0}$ and at generic temperature $T_{0}$. The resulting state can be described by Eq. (9) with external 
field $\mathbf{h}_{0}$, leading, far enough from the critical point, to a Gaussian distribution of the order parameter such as Eq. (11) with $\tau_{0} \sim g_{0} M_{0}^{2}$ where $M_{0}=\left(6\left|\mathbf{h}_{0}\right| / g_{0}\right)^{1 / 3}$. However, within the renormalization-group ( $\mathrm{RG}$ ) approach to the problem it has been shown [36] that $\tau_{0}^{-1}$ is an irrelevant variable in the sense that $\tau_{0}^{-1}$ affects only the correction to the leading long-time scaling behavior we are interested in. In view of that we fix it to the value $\tau_{0}^{-1}=0$ from the very beginning of the calculation. It is important to note that the initial state described by by the Hamiltonian Eq. (11) does not correspond to a low-temperature one in zero magnetic field, unless $n=1$ [30]. In fact, for $n>1$ the transverse modes are critical for all $T<T_{c}$ (Goldstone theorem, see, e.g., [34]) and the proper $H_{0}$ takes the form of Eq. (11) only for the longitudinal mode, whereas the most relevant term (in the RG sense) for the transverse ones would be $\int \mathrm{d}^{d} x\left(\nabla \boldsymbol{\pi}_{0}\right)^{2}$ (where $\left.\boldsymbol{\pi}_{0}(\mathbf{x}, t) \equiv \boldsymbol{\pi}(\mathbf{x}, t=0)\right)$.

In order to account for a non-vanishing mean value of the order parameter $\langle\Phi(\mathbf{x}, t)\rangle=$ $\mathbf{M}(t)$ (we assume that $\mathbf{M}(t)$ stays homogeneous in space after the quench) during the time evolution, it is convenient [33] to write the action (10) in terms of the transverse and longitudinal fluctuations ( $\boldsymbol{\pi}$ and $\sigma$, respectively) around $\mathbf{M}(t)$, i.e.

$$
\Phi(\mathbf{x}, t)=(\sigma(\mathbf{x}, t)+M(t), \boldsymbol{\pi}(\mathbf{x}, t)), \quad \tilde{\Phi}(\mathbf{x}, t)=(\tilde{\sigma}(\mathbf{x}, t), \tilde{\boldsymbol{\pi}}(\mathbf{x}, t)) .
$$

With this parameterization $\langle\sigma(\mathbf{x}, t)\rangle=0$. The problem we shall consider below is therefore the dynamics of fluctuations of the fields $(\sigma(\mathbf{x}, t), \boldsymbol{\pi}(\mathbf{x}, t))$ in the time-dependent "background" provided by $M(t)$. For later convenience we also introduce the scaled magnetization $m(t)$ :

$$
m^{2} \equiv g_{0} \frac{M^{2}}{2}
$$

so that a perturbative expansion in $g_{0}$ leads to a finite value for $m_{0} \equiv m(t=0)$. The resulting action in terms of $\sigma, \tilde{\sigma}, \boldsymbol{\pi}, \tilde{\boldsymbol{\pi}}$ may be written as

$$
S=\int_{0}^{\infty} \mathrm{d} t \int \mathrm{d}^{d} x\left(\mathcal{L}_{0}+\mathcal{L}_{1}+\mathcal{L}_{2}\right)
$$

where

$$
\begin{aligned}
\mathcal{L}_{0}= & -\tilde{\sigma} \Omega \tilde{\sigma}+\tilde{\sigma}\left(\partial_{t}+\Omega\left[-\nabla^{2}+r_{0}+m^{2}(t)\right] \sigma\right) \\
& -\tilde{\boldsymbol{\pi}} \Omega \tilde{\boldsymbol{\pi}}+\tilde{\boldsymbol{\pi}}\left(\partial_{t}+\Omega\left[-\nabla^{2}+r_{0}+\frac{m^{2}(t)}{3}\right]\right) \boldsymbol{\pi}, \\
\mathcal{L}_{1}=\Omega & {\left[\sqrt{2} m(t)\left(\frac{\sqrt{g_{0}}}{2} \tilde{\sigma} \sigma^{2}+\frac{\sqrt{g_{0}}}{6} \tilde{\sigma} \boldsymbol{\pi}^{2}+\frac{\sqrt{g_{0}}}{3} \sigma \tilde{\boldsymbol{\pi}} \boldsymbol{\pi}\right)\right.} \\
+ & \left.\frac{g_{0}}{6} \tilde{\sigma} \sigma^{3}+\frac{g_{0}}{6} \tilde{\sigma} \sigma \boldsymbol{\pi}^{2}+\frac{g_{0}}{6} \tilde{\boldsymbol{\pi}} \boldsymbol{\pi} \boldsymbol{\pi}^{2}+\frac{g_{0}}{6} \sigma^{2} \tilde{\boldsymbol{\pi}} \boldsymbol{\pi}\right], \\
\mathcal{L}_{2}= & \tilde{\sigma}\left(\partial_{t}+\Omega\left[r_{0}+\frac{1}{3} m^{2}(t)\right]\right) \sqrt{\frac{2}{g_{0}}} m(t) \equiv \tilde{\sigma} h_{\mathrm{eff}}(t) .
\end{aligned}
$$

We split up the action $S$ so that $\mathcal{L}_{0}$ is the Gaussian part, $\mathcal{L}_{1}$ contains the interaction vertices and $\mathcal{L}_{2}$ gives the coupling to the effective magnetic field $h_{\text {eff }}(t)$, due to a nonzero $M(t) \propto m(t)$, acting on $\sigma(\mathbf{q}=0, t)$. The presence of $m(t) \neq 0$ breaks the original $O(n)$ 
symmetry of $S$ in the fields $\Phi$ and $\tilde{\Phi}$ (see Eq. (10)), leaving only a reduced $O(n-1)$ symmetry in the transverse field $\boldsymbol{\pi}$ and $\tilde{\boldsymbol{\pi}}$ (to all orders in perturbation theory).

Following standard methods $[34,35]$ the response and correlation functions may be obtained by a perturbative expansion of the functional weight $\mathrm{e}^{-\left(S[\Phi, \tilde{\Phi}]+H_{0}\left[\Phi_{0}\right]\right)}$ in terms of the coupling constant $g_{0}$. The propagators (Gaussian two-point functions of the fields $\sigma$ and $\boldsymbol{\pi}$ in momentum space) are $[36,37]$

$$
\begin{aligned}
&\left\langle\sigma(\mathbf{q}, t) \tilde{\sigma}\left(\mathbf{q}^{\prime}, t^{\prime}\right)\right\rangle_{0}=(2 \pi)^{d} \delta\left(\mathbf{q}+\mathbf{q}^{\prime}\right) R_{\mathbf{q}}^{\sigma}\left(t, t^{\prime}\right), \\
&\left\langle\sigma(\mathbf{q}, t) \sigma\left(\mathbf{q}^{\prime}, t^{\prime}\right)\right\rangle_{0}=(2 \pi)^{d} \delta\left(\mathbf{q}+\mathbf{q}^{\prime}\right) C_{\mathbf{q}}^{\sigma}\left(t, t^{\prime}\right), \\
&\left\langle\boldsymbol{\pi}_{i}(\mathbf{q}, t) \tilde{\boldsymbol{\pi}}_{j}\left(\mathbf{q}^{\prime}, t^{\prime}\right)\right\rangle_{0}=(2 \pi)^{d} \delta_{i j} \delta\left(\mathbf{q}+\mathbf{q}^{\prime}\right) R_{\mathbf{q}}^{\boldsymbol{\pi}}\left(t, t^{\prime}\right), \\
&\left\langle\boldsymbol{\pi}_{i}(\mathbf{q}, t) \boldsymbol{\pi}_{j}\left(\mathbf{q}^{\prime}, t^{\prime}\right)\right\rangle_{0}=(2 \pi)^{d} \delta_{i j} \delta\left(\mathbf{q}+\mathbf{q}^{\prime}\right) C_{\mathbf{q}}^{\boldsymbol{\pi}}\left(t, t^{\prime}\right),
\end{aligned}
$$

where $(q=|\mathbf{q}|)$

$$
\begin{aligned}
R_{\mathbf{q}}^{\sigma}\left(t, t^{\prime}\right) & =\theta\left(t-t^{\prime}\right) \exp \left\{-\Omega\left[\left(q^{2}+r_{0}\right)\left(t-t^{\prime}\right)+\int_{t^{\prime}}^{t} \mathrm{~d} t^{\prime \prime} m^{2}\left(t^{\prime \prime}\right)\right]\right\}, \\
R_{\mathbf{q}}^{\boldsymbol{\pi}}\left(t, t^{\prime}\right) & =\theta\left(t-t^{\prime}\right) \exp \left\{-\Omega\left[\left(q^{2}+r_{0}\right)\left(t-t^{\prime}\right)+\int_{t^{\prime}}^{t} \mathrm{~d} t^{\prime \prime} m^{2}\left(t^{\prime \prime}\right) / 3\right]\right\}, \\
C_{\mathbf{q}}^{\sigma, \boldsymbol{\pi}}\left(t, t^{\prime}\right) & =2 \Omega \int_{0}^{\infty} \mathrm{d} t^{\prime \prime} R_{\mathbf{q}}^{\sigma, \boldsymbol{\pi}}\left(t, t^{\prime \prime}\right) R_{\mathbf{q}}^{\sigma, \boldsymbol{\pi}}\left(t^{\prime}, t^{\prime \prime}\right) .
\end{aligned}
$$

The perturbative expansion can be as usual organized in terms of Feynman diagrams. The response propagators are given by Eq. (22) for the longitudinal mode and by Eq. (23) for the transverse one, and they are represented as directed solid and dashed lines, respectively, where the arrow points towards $t$, the larger of the two times. The correlation propagators are given, instead, by Eq. (24) and are represented as solid and dashed lines for longitudinal and transverse modes, respectively. The interaction vertices are contained in $\mathcal{L}_{1}$ : Four of them are standard time-independent quartic vertices $\propto \Omega g_{0}$ whereas the remaining three are time-dependent cubic ones $\propto \Omega \sqrt{g_{0}} m(t)$, due to a non-zero magnetization $m(t)$. Propagators and vertices are shown in Fig. 1. The contribution of $h_{\text {eff }}(t)$ cancels for a suitable choice of $m(t)$. The evolution equation for the magnetization $m(t)$ can be obtained by solving the equation of motion $\left.\langle\delta S / \delta \tilde{\sigma}(\mathbf{x}, t)\rangle\right|_{\tilde{\sigma}=0}=0$ :

$$
\left[\partial_{t}+\Omega\left(r_{0}+\frac{1}{3} m^{2}(t)\right)\right] m(t)+\Omega \frac{g_{0}}{2} m(t)\left\langle\sigma^{2}+\pi^{2} / 3\right\rangle+\frac{\Omega g_{0}^{3 / 2}}{6 \sqrt{2}}\left\langle\sigma^{3}+\sigma \boldsymbol{\pi}^{2}\right\rangle=0 .
$$

To the lowest order [hereafter $(\mathrm{d} q)=\mathrm{d}^{d} q /(2 \pi)^{d}$ and $\left.\Omega=1\right]$

$$
\begin{aligned}
\left\langle\sigma^{2}(\mathbf{x}, t)\right\rangle & =\int(\mathrm{d} q) C_{\mathbf{q}}^{\sigma}(t, t)+O\left(g_{0}\right), \quad\left\langle\sigma^{3}(\mathbf{x}, t)\right\rangle=O\left(\sqrt{g_{0}}\right), \\
\left\langle\boldsymbol{\pi}^{2}(\mathbf{x}, t)\right\rangle & =(n-1) \int(\mathrm{d} q) C_{\mathbf{q}}^{\boldsymbol{\pi}}(t, t)+O\left(g_{0}\right), \quad\left\langle\sigma \boldsymbol{\pi}^{2}(\mathbf{x}, t)\right\rangle=O\left(\sqrt{g_{0}}\right),
\end{aligned}
$$

and therefore up to one loop and at the critical point $r_{0}=0$, Eq. (25) becomes

$$
0=\partial_{t} m(t)+\frac{m^{3}(t)}{3}+\frac{g_{0}}{2} \int(\mathrm{d} q)\left[C_{\mathbf{q}}^{\sigma}(t, t)+\frac{n-1}{3} C_{\mathbf{q}}^{\boldsymbol{\pi}}(t, t)\right]+O\left(g_{0}^{2}\right) .
$$




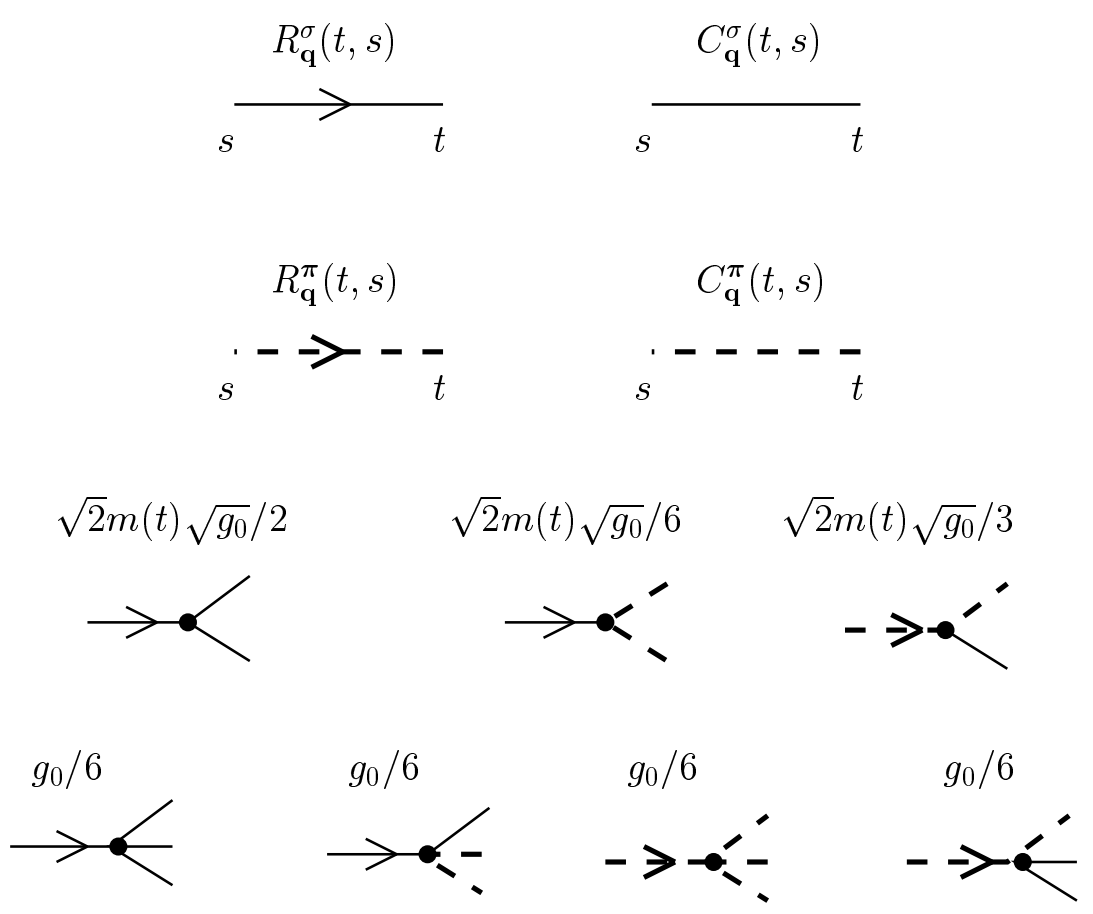

FIG. 1. Elements of the diagrammatic representation of the perturbation theory.

\section{A. Scaling forms}

When a critical system is quenched from the high temperature phase to the critical point response and correlation functions display the scaling forms $[36,6]$

$$
\begin{aligned}
& R_{\mathbf{q}=0}(t, s)=A_{R}(t-s)^{a}(t / s)^{\theta} F_{R}(s / t), \\
& C_{\mathbf{q}=0}(t, s)=A_{C} s(t-s)^{a}(t / s)^{\theta} F_{C}(s / t),
\end{aligned}
$$

where $a=(2-\eta-z) / z, z$ is the dynamical critical exponent, $\eta$ the anomalous dimension of the field, and $\theta$ is a genuine non-equilibrium exponent [36]. $A_{R}$ and $A_{C}$ are non-universal amplitudes which are fixed by the condition $F_{R, C}(0)=1$. With this normalization $F_{R, C}$ are universal and $X^{\infty}$ turns out to be a universal amplitude ratio $X^{\infty}=A_{R} /\left[A_{C}(1-\theta)\right][8,6]$. Although we shall focus mainly on the case $\mathbf{q}=0$, the generalization of these scaling forms to non-vanishing $\mathbf{q}$ amounts to the introduction of an additional scaling variable $y=A_{\Omega} \Omega q^{z}(t-s) . A_{\Omega}$ is a dimensional non-universal constant which can be fixed according to some specified condition. This scaling behavior gets modified when the critical system evolves from a state with $M_{0} \neq 0$. The magnetization scales according to [36]

$$
m(t)=A_{m} m_{0} t^{a+\theta} \mathcal{F}_{M}\left(B_{m} m_{0} t^{\kappa}\right)
$$

where $\kappa=\theta+\beta \delta /(\nu z)-1$ and standard notation for critical exponents has been used. The non-universal amplitudes $A_{m}$ and $B_{m}$ can be determined, e.g., by imposing $\mathcal{F}_{M}(0)=1$ and $\mathcal{F}_{M}^{\prime \prime}(0)=-1$ [30]. From Eq. (31) one sees that the effect of a non-vanishing initial magnetization $m_{0}$ is the introduction of an additional macroscopic time scale $\tau_{m}$ into the problem, i.e., $\tau_{m}=\left(B_{m} m_{0}\right)^{-1 / \kappa}$ and of an additional associated scaling variable $u \equiv t / \tau_{m}$ 
in the scaling forms. This scaling variable, naturally appears in the scaling forms for the response [Eq. (29)] and connected correlation functions [Eq. (30)]:

$$
\begin{aligned}
& R_{\mathbf{q}=0}^{i}(t, s)=A_{R}(t-s)^{a}(t / s)^{\theta} \mathcal{F}_{R}^{i}\left(s / t, B_{m} m_{0} t^{\kappa}\right), \\
& C_{\mathbf{q}=0}^{i}(t, s)=A_{C} s(t-s)^{a}(t / s)^{\theta} \mathcal{F}_{C}^{i}\left(s / t, B_{m} m_{0} t^{\kappa}\right),
\end{aligned}
$$

where no new non-universal amplitudes have been introduced. The index $i=\sigma, \boldsymbol{\pi}$ indicate the longitudinal and transverse fluctuation modes, respectively. The resulting functions $\mathcal{F}_{R}$ and $\mathcal{F}_{C}$ are universal. For $m_{0}=0$ the original $O(n)$ symmetry of the model is restored and therefore transverse and longitudinal response (and correlations) become identical.

According to Eqs. (32) and (33) a non-vanishing mean value of the initial magnetization $m_{0} \neq 0$ affects the scaling properties of the response and correlation function as soon as $B_{m} m_{0} t^{\kappa} \sim 1$ (i.e., $t \sim \tau_{m}$ ) and in particular this happens in the long-time limit we are interested in, characterized by $t \gg s \gg \tau_{m}$. This formally corresponds to the case $m_{0} \rightarrow \infty$, as opposed to the case previously considered, $m_{0}=0$. In this limit one expects the scaling forms (32) and (33) to turn into:

$$
\begin{aligned}
& R_{\mathbf{q}=0}^{\sigma}(t, s)=A_{R}^{\sigma}(t-s)^{a}(t / s)^{\theta_{\sigma}} F_{R}^{\sigma}(s / t), \\
& C_{\mathbf{q}=0}^{\sigma}(t, s)=A_{C}^{\sigma} s(t-s)^{a}(t / s)^{\theta_{\sigma}^{\prime}} F_{C}^{\sigma}(s / t), \\
& R_{\mathbf{q}=0}^{\boldsymbol{\pi}}(t, s)=A_{R}^{\boldsymbol{\pi}}(t-s)^{a}(t / s)^{\theta_{\boldsymbol{\pi}}} F_{R}^{\boldsymbol{\pi}}(s / t), \\
& C_{\mathbf{q}=0}^{\boldsymbol{\pi}}(t, s)=A_{C}^{\boldsymbol{\pi}} s(t-s)^{a}(t / s)^{\theta_{\pi}^{\prime}} F_{C}^{\boldsymbol{\pi}}(s / t) .
\end{aligned}
$$

The non-universal constants $A_{R, C}^{i}$ are fixed by requiring $F_{R, C}^{i}(0)=1$, where $F_{R, C}^{i}(x)$ are universal functions related to the large- $v$ behavior of $\mathcal{F}_{R, C}^{i}(x, v)$. The exponents $\theta_{i}$ and $\bar{\theta}_{i}^{\prime}$ are different from $\theta$. In particular it is not obvious, a priori, whether $\theta_{i}$ and $\theta_{i}^{\prime}$ should be expected to be the same, if they are novel exponents - as $\theta$ - or just combinations of known ones.

In Ref. [30] we showed, for the Ising model (i.e., the $O(n)$ model with $n=1$ ), that

$$
\theta_{\sigma}=\theta_{\sigma}^{\prime}=-\frac{\beta \delta}{\nu z}
$$

The RG proof of Ref. [30] made no use of the scalar nature of the order parameter and holds for any purely dissipative model (i.e., with Model A dynamics).

For the transverse modes, it has been argued in Ref. [24] that

$$
\theta_{\boldsymbol{\pi}}=\theta_{\boldsymbol{\pi}}^{\prime}=-\frac{\beta}{\nu z}
$$

A fundamental difference between the quench from a disordered and a magnetized state emerges: In the latter case the aging properties and the non-equilibrium behavior of both longitudinal and transverse modes are completely described in terms of equilibrium exponents.

As a consequence of the scaling forms Eqs. (34), (35), (36), and (37) the two limiting FDR are

$$
X_{\sigma}^{\infty}\left(m_{0} \neq 0\right)=\frac{A_{R}^{\sigma}}{A_{C}^{\sigma}\left(1-\theta_{\sigma}\right)}, \quad X_{\boldsymbol{\pi}}^{\infty}\left(m_{0} \neq 0\right)=\frac{A_{R}^{\boldsymbol{\pi}}}{A_{C}^{\boldsymbol{\pi}}\left(1-\theta_{\boldsymbol{\pi}}\right)},
$$

which turn out to be universal amplitude ratios [30]. 


\section{GAUSSIAN APPROXIMATION}

The starting point for the analysis of the dynamics resulting from the action $S$ [see Eqs. (10) and (14)] is the Gaussian approximation in which one neglects all the terms which are of degree higher than 2 in the fields $\boldsymbol{\pi}$ and $\sigma$, contained in $\mathcal{L}_{1}$. The result of such an approximation is exact if the space dimensionality is greater than the upper critical dimension $d_{u}=4[34,6]$.

Within this approximation, the equation of motion (28) at the critical point $r_{0}=0$ becomes

$$
\partial_{t} m_{G}(t)=-\frac{1}{3} m_{G}^{3}(t)
$$

( $G$ stands for Gaussian) whose solution is

$$
m_{G}^{2}=\frac{1}{\frac{2}{3} t+\frac{1}{m_{0}^{2}}}=(2 t / 3)^{-1}\left(1+\frac{3}{2 m_{0}^{2} t}\right)^{-1} .
$$

This expression agrees with the scaling behavior (31), given that $\theta=a=0$ and $\kappa=\frac{1}{2}$ within the Gaussian model. The non-universal amplitudes appearing in Eq. (31) are $A_{m}=1$ and $B_{m}=\sqrt{2 / 3}$, leading to $\tau_{m}=\left(B_{m} m_{0}\right)^{-1 / \kappa}=3 /\left(2 m_{0}^{2}\right)$ and $m_{G}(t)=m_{0}\left(1+t / \tau_{m}\right)^{-1 / 2}$. For $t \gg \tau_{m}, m_{G}(t) \sim(2 t / 3)^{-1 / 2}$ and a nonzero $m_{0}^{-1}$ affects only the corrections to this leading long-time behavior. In this sense $m_{0}^{-1}$ is irrelevant for long times.

Gaussian response and correlation functions (see Eqs. (22) and (24)) do not depend on $n$ and therfore the longitudinal ones are the same as those of the Ising model studied in Ref. [30], which the reader is referred to for details. We recall here that the Gaussian FDR turns out to be $X_{\sigma}^{\infty}=4 / 5$ [30]. For the transverse critical response and correlation functions, Eqs. (23) and (24) give $(t>s)$

$$
\begin{aligned}
& R_{\mathbf{q}}^{\boldsymbol{\pi}, 0}(t, s)=\mathrm{e}^{-\mathbf{q}^{2}(t-s)-\int_{s}^{t} \mathrm{~d} t^{\prime} m_{G}^{2}\left(t^{\prime}\right) / 3}=\left(\frac{s+\tau_{m}}{t+\tau_{m}}\right)^{1 / 2} \mathrm{e}^{-\mathbf{q}^{2}(t-s)}, \\
& C_{\mathbf{q}, 0}^{\boldsymbol{\pi}, 0}(t, s)=2 \int_{0}^{s} \mathrm{~d} t^{\prime} R_{\mathbf{q}}^{\boldsymbol{\pi}, 0}\left(t, t^{\prime}\right) R_{\mathbf{q}}^{\boldsymbol{\pi}, 0}\left(s, t^{\prime}\right)=2 \frac{\mathrm{e}^{-\mathbf{q}^{2}(t+s)}}{\left[\left(t+\tau_{m}\right)\left(s+\tau_{m}\right)\right]^{1 / 2}} \int_{0}^{s} \mathrm{~d} t^{\prime}\left(t^{\prime}+\tau_{m}\right) \mathrm{e}^{2 \mathbf{q}^{2} t^{\prime}} .
\end{aligned}
$$

In particular, for $\mathbf{q}=0$ one finds

$$
\begin{aligned}
& R_{\mathbf{q}=0}^{\boldsymbol{\pi}, 0}(t, s)=\left(\frac{s+\tau_{m}}{t+\tau_{m}}\right)^{1 / 2} \\
& C_{\mathbf{q}=0}^{\boldsymbol{\pi}, 0}(t, s)=\frac{\left(s+\tau_{m}\right)^{2}-\tau_{m}^{2}}{\left[\left(s+\tau_{m}\right)\left(t+\tau_{m}\right)\right]^{1 / 2}} .
\end{aligned}
$$

Comparing these results (for $\tau_{m}=0$ ), with the scaling forms (36) and (37) we can identify $z=2, a=0, \theta_{\boldsymbol{\pi}}=-1 / 2$ in agreement with standard mean-field exponents $(\nu=\beta=1 / 2$ and $\eta=0$ ) and determine $A_{R}^{\boldsymbol{\pi}}=1, A_{C}^{\boldsymbol{\pi}}=1, F_{R}^{\boldsymbol{\pi}}(x)=1$, and $F_{C}^{\boldsymbol{\pi}}(x)=1$. Accordingly, the asymptotic FDR is given by (see Eq. (40))

$$
X_{\boldsymbol{\pi}}^{\infty}=\frac{A_{R}^{\boldsymbol{\pi}}}{A_{C}^{\boldsymbol{\pi}}\left(1-\theta_{\boldsymbol{\pi}}\right)}=\frac{2}{3},
$$




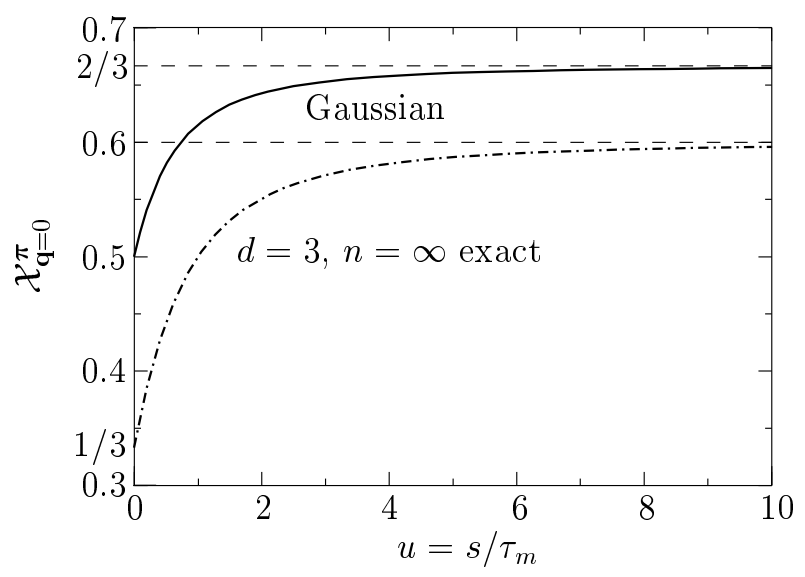

(a)

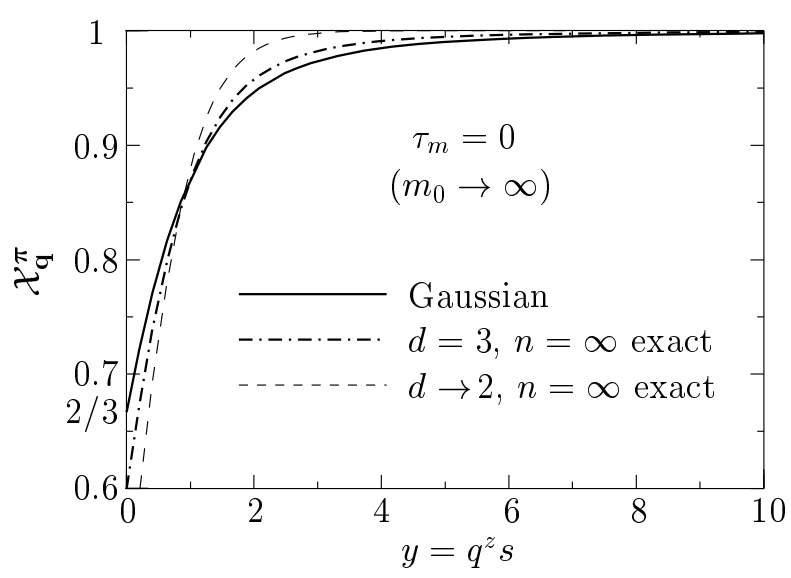

(b)

FIG. 2. Transverse FDR $\mathcal{X}_{\mathbf{q}}^{\boldsymbol{\pi}}$ within the Gaussian approximation (solid line) and in the exactly solvable limit $n \rightarrow \infty$ for $d=3$ (dash-dotted line) and $d \rightarrow 2$ (dotted line in the right panel). Left: $\mathcal{X}_{\mathbf{q}=0}^{\boldsymbol{\pi}}$ as a function of $s / \tau_{m}$. Right: $\mathcal{X}_{\mathbf{q}}^{\boldsymbol{\pi}}$ for $\tau_{m}=0$ as a function of $q^{z} s$.

which differs from the corresponding quantity for the longitudinal mode $X_{\sigma}^{\infty}=4 / 5$. To our knowledge this is the first case in which the asymptotic FDR of two local quantities differ already within the Gaussian approximation. In fact in Ref. [20] we showed that, for a quench from the disordered phase to the critical point, the Gaussian $X_{\mathcal{O}}^{\infty}$ is always $1 / 2$, independently of the specific quantity $\mathcal{O}$ it refers to. As a consequence an effective temperature $T_{\text {eff }} \equiv T_{c} / X^{\infty}$ is not well-defined even within the Gaussian approximation for the quench from a magnetized state $[38,39,20]$.

Let us discuss in more detail the effect of a non-zero magnetization on the FDR

$$
\mathcal{X}_{\mathbf{q}}^{\boldsymbol{\pi}}(t, s) \equiv \frac{R_{\mathbf{q}}^{\boldsymbol{\pi}, 0}(t, s)}{\partial_{s} C_{\mathbf{q}}^{\boldsymbol{\pi}, 0}(t, s)} .
$$

From Eqs. (45) and (46) one finds, as a function of $u=s / \tau_{m}$,

$$
\mathcal{X}_{\mathbf{q}=0}^{\boldsymbol{\pi}}(u)=\frac{2}{3}\left[1+\frac{1}{3}(1+u)^{-2}\right]^{-1},
$$

which is actually independent of $t$ (a typical property of the Gaussian approximation) and increases monotonically from the value $1 / 2$ for $u=0$, to the value $2 / 3$ for $u \rightarrow \infty$. Accordingly, whenever $m_{0}$ is non zero, the transverse FDR approaches $2 / 3$ in the long-time limit (and its value is indeed independent of the actual $m_{0} \neq 0$ ) whereas the FDR is equal to $1 / 2$ for $m_{0}=0$, i.e., for quenches from a disordered initial state. The plot of $\mathcal{X}_{\mathbf{q}=0}^{\boldsymbol{\pi}}(s, t)$ as a function of $u=s / \tau_{m}$ is reported in Fig. 2(a). In the case $\tau_{m}=\infty$ (corresponding to $\left.m_{0}=0\right)$ it was found that the fluctuation mode responsible for aging is the homogeneous one $\mathbf{q}=0$, given that for $\mathbf{q} \neq 0$ and long times $\mathcal{X}_{\mathbf{q}}^{\sigma}=\mathcal{X}_{\mathbf{q}}^{\boldsymbol{\pi}}=1[3,6]$. The same conclusion was drawn for the (longitudinal) fluctuations of the Ising model with $\tau_{m}=0$ (corresponding to $m_{0}=\infty$ ) [30]. Now for the transverse modes one finds, from Eqs. (43) and (44), as a function of $y=q^{2} s$,

$$
\mathcal{X}_{\mathbf{q}}^{\boldsymbol{\pi}}(y)=\frac{4 y^{2}}{1+4 y^{2}-\mathrm{e}^{-2 y}(1+2 y)}
$$


which monotonically increases from the value $2 / 3$ at $y=0$ to 1 for $y \rightarrow \infty$ [see Fig. 2(b)], as observed in all the other cases studied so far.

\section{DYNAMICS OF THE TRANSVERSE MODES IN THE LIMIT $N \rightarrow \infty$}

Critical transverse response and correlation functions can be analytically calculated in the limit of large number of components of the order parameter. In fact, assuming that $g_{0} \equiv g / n$ (with $g$ independent of $n$ ), the action (14) for large $n$ may be written as $(\Omega=1)$

$$
S(\boldsymbol{\pi}, \tilde{\boldsymbol{\pi}} ; m)=\int \mathrm{d}^{d} x \mathrm{~d} t\left\{-\tilde{\boldsymbol{\pi}} \tilde{\boldsymbol{\pi}}+\tilde{\boldsymbol{\pi}}\left[\partial_{t}+\left(-\nabla^{2}+r_{0}+\frac{m^{2}(t)}{3}\right)\right] \boldsymbol{\pi}+\frac{g}{6} \frac{1}{n} \tilde{\boldsymbol{\pi}} \boldsymbol{\pi} \boldsymbol{\pi}^{2}\right\}+O\left(n^{0}\right),
$$

where $m(t)$ satisfies the equation of motion

$$
\left(\partial_{t}+r_{0}+\frac{m^{2}(t)}{3}+\frac{g}{6} \frac{\left\langle\boldsymbol{\pi}^{2}\right\rangle}{n}+O\left(n^{-1}\right)\right) m(t)=0 .
$$

It is evident that only transverse modes contribute to the leading term of the action. On the one hand, this makes the calculation easier but on the other the behavior of the longitudinal mode cannot be determined without including the subleading $O\left(n^{0}\right)$ terms in the action.

To proceed further we self-consistently factorize the fourth order interaction term according to $[34,42]$

$$
\frac{1}{n} \tilde{\boldsymbol{\pi}} \boldsymbol{\pi} \boldsymbol{\pi}^{2} \rightarrow C_{\mathbf{x}=0}^{\boldsymbol{\pi}}(t, t) \tilde{\boldsymbol{\pi}} \boldsymbol{\pi} .
$$

The model resulting from this factorization is Gaussian with a time-dependent temperature:

$$
\hat{r}(t)=r_{0}+\frac{g}{6} C_{\mathbf{x}=0}^{\boldsymbol{\pi}}(t, t)+\frac{m^{2}(t)}{3} .
$$

The response and correlation functions are $\left(\tau_{0}^{-1}=0\right)$

$$
\begin{aligned}
& R_{\mathbf{q}}^{\boldsymbol{\pi}}(t, s)=\exp \left\{-\int_{s}^{t} \mathrm{~d} t^{\prime}\left[q^{2}+\hat{r}\left(t^{\prime}\right)\right]\right\}, \\
& C_{\mathbf{q}}^{\boldsymbol{\pi}}(t, s)=2 \int_{0}^{s} \mathrm{~d} t^{\prime} R_{\mathbf{q}}^{\boldsymbol{\pi}}\left(t, t^{\prime}\right) R_{\mathbf{q}}^{\boldsymbol{\pi}}\left(s, t^{\prime}\right),
\end{aligned}
$$

with the self-consistency condition $\left[\hat{r}_{\infty} \equiv \hat{r}(t \rightarrow \infty)\right]$

$$
\hat{r}(t)=\hat{r}_{\infty}+\frac{g}{6} \int^{\Lambda}(\mathrm{d} q)\left[C_{\mathbf{q}}^{\boldsymbol{\pi}}(t, t)-C_{\mathbf{q}}^{\boldsymbol{\pi}}(\infty, \infty)\right]+\frac{m^{2}(t)}{3},
$$

where $C_{\mathbf{q}}^{\boldsymbol{\pi}}(\infty, \infty)=1 /\left(q^{2}+\hat{r}_{\infty}\right)$ is the equilibrium correlation function and $\Lambda$ is a largemomentum cut-off. The equation of motion (52) becomes

$$
\partial_{t} m(t)+\hat{r}(t) m(t)=0,
$$


which is solved by

$$
m(t)=m_{0} \exp \left[-\int_{0}^{t} \mathrm{~d} t^{\prime} \hat{r}\left(t^{\prime}\right)\right] .
$$

It is possible to cast the previous equations in a very convenient form by introducing the function

$$
h\left(2 \Lambda^{2} t\right)=\frac{1}{2} \exp \left[2 \int_{0}^{t} \mathrm{~d} t^{\prime} \hat{r}\left(t^{\prime}\right)\right]
$$

( $h$ corresponds to the function $g$ usually introduced in the literature on the dynamics of the spherical model $[8,26])$ in terms of which

$$
\begin{aligned}
m(t) & =m_{0}\left[2 h\left(2 \Lambda^{2} t\right)\right]^{-1 / 2}, \\
R_{\mathbf{q}}^{\boldsymbol{\pi}}(t, s) & =\mathrm{e}^{-\mathbf{q}^{2}(t-s)}\left[\frac{h\left(2 \Lambda^{2} s\right)}{h\left(2 \Lambda^{2} t\right)}\right]^{1 / 2},
\end{aligned}
$$

and the expression for $C_{\mathbf{q}}^{\boldsymbol{\pi}}$ readily follows. Accordingly, once we know the function $h(x)$ for a generic initial magnetization, the dynamics of transverse modes is completely determined. The details of the computation of $h(x)$ are reported in Appendix A. The final result for the critical case $\left(\hat{r}_{\infty}=0\right), 2<d<4(\epsilon \equiv 4-d)$, and in the scaling regime $t \gg\left(2 \Lambda^{2}\right)^{-1}$ is

$$
h\left(2 \Lambda^{2} t \gg 1\right)=\frac{1}{u^{*}} \frac{\sin (\pi(d-2) / 2)}{\pi \Gamma((d-2) / 2)}\left(2 \Lambda^{2} t\right)^{-\epsilon / 2}\left[1+\frac{4}{3(d-2)} m_{0}^{2} t\right],
$$

where $u^{*}=g^{*} N_{d} /\left(6 \Lambda^{\epsilon}\right)$ is the fixed-point value of the four-point coupling (see Appendix A) and corrections to the leading scaling behavior have been neglected.

According to Eq. (61), the magnetization is given by

$$
m(t)=m_{0}\left[u^{*} \frac{\pi \Gamma((d-2) / 2)}{2 \sin (\pi(d-2) / 2)}\right]^{1 / 2}\left(2 \Lambda^{2} t\right)^{\epsilon / 4}\left[1+\frac{4}{3(d-2)} m_{0}^{2} t\right]^{-1 / 2},
$$

which can be cast in the scaling form Eq. (31) with (we recall that $a=0, \beta \delta /(\nu z)=$ $(d+2) / 4[34], \theta=\epsilon / 4[36]$, and therefore $\kappa=1 / 2)$

$$
A_{m}=\left[u^{*} \frac{\pi \Gamma((d-2) / 2)}{2 \sin (\pi(d-2) / 2)}\right]^{1 / 2}\left(2 \Lambda^{2}\right)^{\epsilon / 4}, \quad B_{m}=\left[\frac{4}{3(d-2)}\right]^{1 / 2},
$$

and

$$
\mathcal{F}_{M}(v)=\left(1+v^{2}\right)^{-1 / 2} .
$$

The time scale associated to the initial magnetization is therefore

$$
\tau_{m}=\left(B_{m} m_{0}\right)^{-1 / \kappa}=\frac{3}{4}(d-2) m_{0}^{-2} .
$$

The response function follows from Eqs. (62) and (63):

$$
R_{\mathbf{q}}^{\boldsymbol{\pi}}(t, s)=\mathrm{e}^{-\mathbf{q}^{2}(t-s)}\left(\frac{t}{s}\right)^{\epsilon / 4}\left[\frac{1+s / \tau_{m}}{1+t / \tau_{m}}\right]^{1 / 2}
$$


which displays (for $\mathbf{q}=0$ ) the expected scaling behaviors (29), (32), and (36), for $\tau_{m}=\infty$, generic $\tau_{m}$, and $\tau_{m}=0$, respectively. [We recall that $\theta_{\boldsymbol{\pi}}=\theta_{\boldsymbol{\pi}}^{\prime}=-\beta /(\nu z)=-1 / 2+\epsilon / 4$.] In particular, comparing for $\tau_{m}=0$ to Eq. (36), one finds $A_{R}^{\boldsymbol{\pi}}=1, F_{R}^{\boldsymbol{\pi}}(x) \equiv 1$. The correlation function $C_{\mathbf{q}}^{\boldsymbol{\pi}}(t, s)$ can be easily computed from Eq. (68) via Eq. (56). In particular, for $\mathbf{q}=0$ one finds

$$
C_{\mathbf{q}=0}^{\boldsymbol{\pi}}(t, s)=\frac{4}{d} s\left(\frac{t}{s}\right)^{\epsilon / 4}\left[\frac{1+s / \tau_{m}}{1+t / \tau_{m}}\right]^{1 / 2}\left[1+\frac{2}{d-2} \frac{1}{1+s / \tau_{m}}\right],
$$

which displays also the expected scaling behaviors (30), (33), and (37), for $\tau_{m}=\infty$, generic $\tau_{m}$, and $\tau_{m}=0$, respectively. In particular Eq. (36) holds with $A_{C}^{\boldsymbol{\pi}}=4 / d$ and $F_{C}^{\boldsymbol{\pi}}(x) \equiv 1$.

Equations (68) and (69) allow the computation of the FDR:

$$
\mathcal{X}_{\mathbf{q}=0}^{\boldsymbol{\pi}}(u)=\frac{d}{d+2}\left[1+\frac{4}{d^{2}-4} \frac{1}{(1+u)^{2}}\right]^{-1},
$$

where $u \equiv s / \tau_{m}$. For $m_{0}=0$ (i.e., $s \ll \tau_{m} \propto m_{0}^{-2}$ ) we recover the well-known result $X^{\infty}=1-2 / d[8,20]$, whereas for $m_{0} \neq 0$ the long-time asymptotic value for $s \gg \tau_{m}$ is given by

$$
X_{\pi}^{\infty}(n=\infty)=\frac{d}{d+2} .
$$

Close to $d=2$ this expression becomes

$$
X_{\pi}^{\infty}(n=\infty)=\frac{1}{2}+\frac{1}{8} \tilde{\epsilon}+O\left(\tilde{\epsilon}^{2}\right)
$$

where $\tilde{\epsilon}=d-2$, in agreement with the limit $n \rightarrow \infty$ of the result of Ref. [24] (see Eq. (18) therein):

$$
X^{\infty}=\frac{1}{2}+\frac{1}{8} \frac{n-1}{n-2} \tilde{\epsilon}+O\left(\tilde{\epsilon}^{2}\right) .
$$

In passing we note that Eq. (71) coincides with the FDR derived in Ref. [26] for local spin observables in the spherical model (see Eq. (8.12) therein).

A plot of Eq. (71) is provided as a dashed line in Fig. 6 (right panel) together with the result of the expansion (73) for $n=3$ and $n=\infty$ (denoted by $n=3[\tilde{\epsilon}]$ and $n=\infty[\tilde{\epsilon}]$, respectively). As in the Gaussian case, $\mathcal{X}_{\mathbf{q}=0}^{\boldsymbol{\pi}}(u)$ can be used to describe the crossover from magnetized to non-magnetized initial state and the scaling function resembles closely Eq. (49), - derived for the Gaussian model and corresponding to the case $d>4$ - the only difference being the values at $u=0$ and for $u \rightarrow \infty$ and the actual expression for $\tau_{m}$ as a function of $m_{0}$. In Fig. 2(a) we compare the exact scaling function for $d=3$ (see Eq. (70)) to the Gaussian one (Eq. (49), formally $d=4$ ). In both cases $\mathcal{X}_{\mathbf{q}=0}^{\boldsymbol{\pi}}$ increases monotonically from the value $1-2 / d$ for $u=0$ to $d /(d+2)$ for $u \rightarrow \infty$.

As in the case of the Gaussian approximation (see Sec. III), we consider the momentumdependence of the FDR. As a function of the two scaling variables $y=q^{z} s=q^{2} s$ and $u=s / \tau_{m}$ (where $\tau_{m}$ is given in Eq. (67)) one finds, from Eqs. (68) and (56):

$$
\mathcal{X}_{\mathbf{q}}^{\boldsymbol{\pi}}=\frac{1}{2}\left[1-\left(\frac{d-2}{4}+y-\frac{1}{2} \frac{1}{1+u}\right) \int_{0}^{1} \mathrm{~d} x(1-x)^{(d-4) / 2}\left(1-\frac{u}{1+u} x\right) \mathrm{e}^{-2 x y}\right]^{-1}
$$




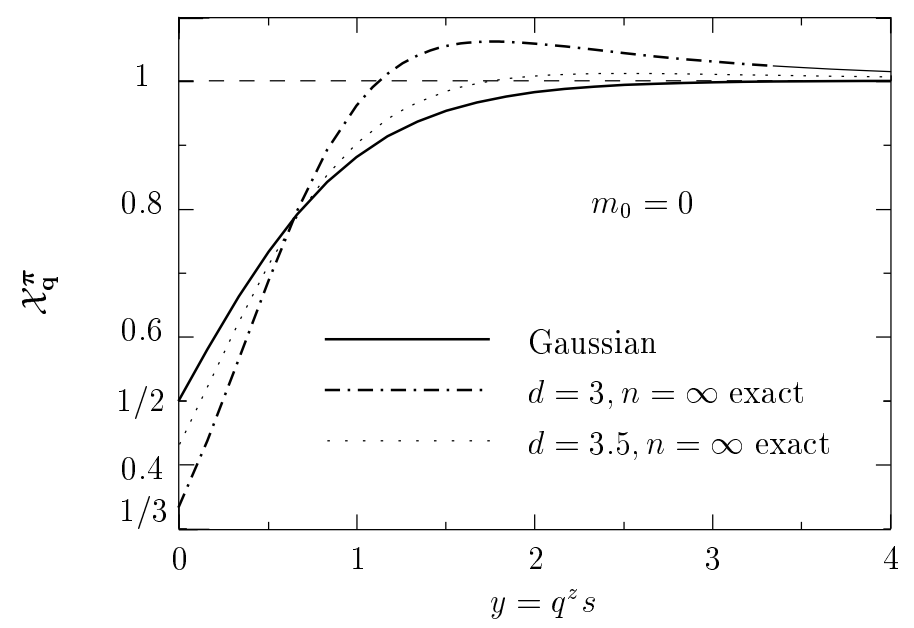

FIG. 3. Non-monotonic behavior of $\mathcal{X}_{\mathbf{q}}^{\boldsymbol{\pi}}$ as a function of $y=q^{z} s$ and different values of $2<d<4$ for the exactly solvable case $n \rightarrow \infty$ of the $O(n)$ model relaxing from a disordered initial state $\left(m_{0}=0\right)$. The Gaussian result corresponds to $d>4$.

from which one recovers Eq. (70) for $y=0$ and the Gaussian result (50) for $d=4$ and $u \rightarrow \infty$, as expected. Taking into account that $\int_{0}^{1} \mathrm{~d} x(1-x)^{-\epsilon / 2} \mathrm{e}^{-2 y x}=1 /(2 y)+\epsilon /\left(8 y^{2}\right)+$ $\epsilon(2+\epsilon) /\left(32 y^{3}\right)+O\left(y^{-4}\right)$ one easily determines the behavior of $\mathcal{X}_{\mathbf{q}}^{\boldsymbol{\pi}}$ for $y \gg 1$ :

$$
\mathcal{X}_{\mathbf{q}}^{\boldsymbol{\pi}}=1-\frac{1}{4 y^{2}}\left[\left(\frac{u}{1+u}\right)^{2}-\frac{\epsilon}{2}\right]+O\left(y^{-3}\right) .
$$

Accordingly, for $y=q^{2} s \gg 1$ the FDR converges to the asymptotic value 1 , as heuristically expected on the basis of the fact that the mode actually responsible for the aging behavior is the homogeneous one $\mathbf{q}=0$. We note also that this asymptotic value is approached from above for $0 \leq u<1 /(\sqrt{2 / \epsilon}-1)$ and from below for $u>1 /(\sqrt{2 / \epsilon}-1)$. Given that $\mathcal{X}_{\mathbf{q}}^{\boldsymbol{\pi}}<1$ for $y=0, \mathcal{X}_{\mathbf{q}}^{\boldsymbol{\pi}}$ has a non-monotonic behavior as a function of $y$ for fixed $0 \leq u<1 /(\sqrt{2 / \epsilon}-1)$ and in particular for $u=0$, corresponding to an initially disordered configuration. This non-monotonicity disappears in the Gaussian limit $d \rightarrow 4$ and was already noticed at the first order in the $\epsilon$-expansion for the relaxation of the $O(n)$ model from a disordered state (see Fig. 1 in Ref. [7] and Ref. [3] for details). In fact, the expansion in $\epsilon=4-d$ of Eq. (74) for $u=0$ agrees with the limit $n \rightarrow \infty$ of the results presented in Ref. [3] (cp. Eq. (3.14) therein). In Fig. 3 we provide a plot of Eq. (74), as a function of $y=q^{2} s$ for $m_{0}=0$ and $d=3,3.5$, and 4 , the latter corresponding to the Gaussian model. The non-monotonic behavior is still quite pronounced for $d=3$ and the overshoot decreases upon increasing the dimensionality $d$ (cf. the dotted line corresponding to the unphysical dimension $d=3.5$, reported for comparison), while shifting towards larger values of $y$. In the opposite limit, i.e., $u \rightarrow \infty$ (corresponding to $\tau_{m}=0$ ), a monotonic increase of $\mathcal{X}_{\mathbf{q}}^{\boldsymbol{\pi}}$ as a function of $y=q^{2} s$ is expected. In Fig. 2(b) we compare the behavior predicted in this case by Eq. (74) (with $u \rightarrow \infty$ ) for $d=3$, to the Gaussian result (50) (formally, $d=4$ ). We also indicate the limiting curve for $d \rightarrow 2$. In these cases $\mathcal{X}_{\mathbf{q}}^{\boldsymbol{\pi}}$ increases monotonically from its initial value, given by $d /(d+2)$, to the asymptotic value 1 , which is approached according to Eq. (75). 


\section{ONE-LOOP FLUCTUATION-DISSIPATION RATIOS}

In the previous sections we have discussed the non-equilibrium behavior of models whose action is essentially quadratic in the fields (either because non-Gaussian terms were neglected or turned into effective Gaussian ones). In this section we account for the effects of nonGaussian fluctuations within the perturbation theory up to one-loop (i.e., $O(\epsilon)$ in the $\epsilon$ expansion where $\epsilon=4-d$ ). In particular we compute the longitudinal and transverse correlation and response functions for a quench from a state with initial magnetization $m_{0} \rightarrow \infty$ to the critical point. As we have argued from general scaling arguments, a finite $m_{0}$ gives only corrections to the leading long-time behavior. The first step in this calculation is the solution of the one-loop equation of motion, presented in the next subsection. Then we calculate the connected (longitudinal and transverse) correlation and response functions (and the associated FDRs) in the following subsections, reporting the relevant details in Appendix B.

For future reference we recall that the standard static exponents $\beta, \delta, \nu$ of the universality class we are presently interested in are such that $\beta / \nu=(d-2+\eta) / 2, \delta=(d+2-\eta) /(d-2+\eta)$ with $\eta=O\left(\epsilon^{2}\right)$ and $z=O\left(\epsilon^{2}\right)$ (see, e.g., [34]), and therefore [cp. Eqs. (38) and (39)]

$$
a=O\left(\epsilon^{2}\right), \quad \theta_{\sigma}=-\frac{\beta \delta}{\nu z}=-\frac{3}{2}+\frac{\epsilon}{4}+O\left(\epsilon^{2}\right), \text { and } \quad \theta_{\boldsymbol{\pi}}=-\frac{\beta}{\nu z}=-\frac{1}{2}+\frac{\epsilon}{4}+O\left(\epsilon^{2}\right) .
$$

\section{A. The equation of motion and its solution}

At one-loop level the tadpole contributions to the equation of motion (28) are

$$
\begin{aligned}
& I_{\sigma}(t)=\int(\mathrm{d} q) C_{\mathbf{q}}^{\sigma, 0}(t, t)=2 N_{d} r_{\sigma} t^{1-d / 2}, \\
& I_{\boldsymbol{\pi}}(t)=\int(\mathrm{d} q) C^{\boldsymbol{\pi}, 0}(t, t)_{\mathbf{q}}=2 N_{d} r_{\boldsymbol{\pi}} t^{1-d / 2},
\end{aligned}
$$

where $N_{d}=2 /\left[(4 \pi)^{d / 2} \Gamma(d / 2)\right]$, and

$$
\begin{aligned}
& r_{\sigma}=\frac{96}{(8 \pi)^{d / 2}(8-d)(6-d)(4-d)(2-d) N_{d}} \\
& r_{\boldsymbol{\pi}}=\frac{4}{(8 \pi)^{d / 2}(4-d)(2-d) N_{d}} .
\end{aligned}
$$

Accordingly, the one-loop equation of motion is [see Eq. (28)]

$$
\begin{aligned}
0 & =\partial_{t} m+\frac{1}{3} m^{3}+g_{0} N_{d}\left(r_{\sigma}+\frac{n-1}{3} r_{\boldsymbol{\pi}}\right) t^{1-d / 2} m \equiv+O\left(g_{0}^{2}\right) \\
& \equiv \partial_{t} m+\frac{1}{3} m^{3}+\tilde{g}_{0} r_{d} t^{1-d / 2} m+O\left(\tilde{g}_{0}^{2}\right)
\end{aligned}
$$

where $\tilde{g}_{0} \equiv N_{d} g_{0}$ and

$$
r_{d} \equiv r_{\sigma}+\frac{n-1}{3} r_{\boldsymbol{\pi}}
$$




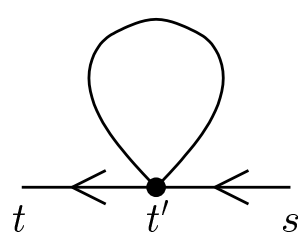

(1)

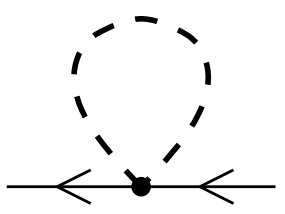

(3)

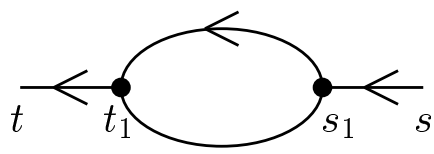

$(2)$

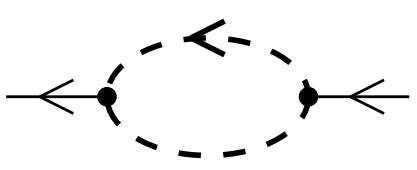

(4)

FIG. 4. One-loop diagrams contributing to the longitudinal response function $R_{\mathbf{q}}^{\sigma}(t, s)$. The corresponding expressions (reported in Appendix B 1) are indicated by $I_{i}(t, s), i=1, \ldots 4$.

which is solved by

$$
m(t)=\sqrt{\frac{3}{2 t}}\left[1-\frac{\tilde{g}_{0}}{3-d / 2} r_{d} t^{\epsilon / 2}\right]+O\left(\tilde{g}_{0}^{2}\right) .
$$

Expanding in $\epsilon=4-d$, one obtains

$$
m(t)=\left(1-\tilde{g}_{0} \frac{c_{-1}}{\epsilon}\right) \sqrt{\frac{3}{2 t}}\left[1-\tilde{g}_{0}\left(\frac{c_{-1}}{2} \ln t+c_{0}-\frac{c_{-1}}{2}\right)+O\left(\epsilon^{2}, \epsilon \tilde{g}_{0}, \tilde{g}_{0}^{2}\right)\right],
$$

where $c_{-1}=-(n+8) / 12$ and $c_{0}=9 / 16-(n+8)\left(\ln 2+\gamma_{E}\right) / 24$ are the coefficients of the expansion of $r_{d}=c_{-1} / \epsilon+c_{0}+O(\epsilon)$. The dimensional pole $\sim 1 / \epsilon$ can be cancelled out by a proper renormalization of the bare magnetization (see, e.g., Ref. [34,32,33,30] for details). Once the renormalized $m(t)$ is expressed in terms of $\tilde{g}=\tilde{g}_{0}+O\left(\tilde{g}_{0}^{2}\right)$, its scaling behavior at the RG fixed point $\tilde{g}^{*}=6 \epsilon /(n+8)+O\left(\epsilon^{2}\right)$ clearly shows up: $m(t) \sim t^{-\varsigma}+O\left(\epsilon^{2}\right)$ with $\varsigma=1 / 2\left(1+c_{-1} \tilde{g}^{*}\right)+O\left(\epsilon^{2}\right)=\beta /(\nu z)$.

\section{B. The Longitudinal response function}

At one-loop order the expression for the response function gets modified because of the one-loop term contributing to the magnetization $m(t)$ in Eq. (85), and therefore

$$
\begin{aligned}
R_{\mathbf{q}}^{\sigma, 0}(t, s) & =\exp \left[-q^{2}(t-s)-\int_{s}^{t} \mathrm{~d} t^{\prime} m^{2}\left(t^{\prime}\right)\right] \\
& =\left(\frac{s}{t}\right)^{3 / 2}\left[1+\tilde{g}_{0} \frac{3}{1+\epsilon / 2} r_{d} \frac{t^{\epsilon / 2}-s^{\epsilon / 2}}{\epsilon / 2}+O\left(\tilde{g}_{0}^{2}\right)\right] \mathrm{e}^{-q^{2}(t-s)}
\end{aligned}
$$


In addition, the interaction vertices of $\mathcal{L}_{1}$ [see Eq. (16)] yield four contributions which are depicted in Fig. 4. In terms of those diagrams the response function is given by

$$
R_{\mathbf{q}}^{\sigma}(t, s)=R_{\mathbf{q}}^{\sigma, 0}(t, s)-\frac{g_{0}}{2} I_{1}-g_{0} \frac{n-1}{6} I_{3}+g_{0} I_{2}+g_{0} \frac{n-1}{9} I_{4}+O\left(g_{0}^{2}\right) .
$$

The relevant zero-momentum integrals $I_{i}, i=1, \ldots, 4$, have been calculated in Appendix B 1 . Collecting the corresponding expressions we obtain, in terms of the renormalized coupling $\tilde{g}$ and of the ratio $x \equiv s / t(0 \leq x \leq 1)$,

$$
R_{\mathbf{q}=0}^{\sigma}(t, s)=x^{3 / 2}\left[1+\tilde{g}\left\{-\frac{n+8}{24} \ln x+\left(\frac{\pi^{2}}{4}-\frac{61+2 n}{24}+f_{R}^{\sigma}(x)\right)\right]\right\}+O\left(\tilde{g}^{2}, \epsilon \tilde{g}\right)
$$

where

$$
f_{R}^{\sigma}(x)=\frac{26+n}{12}\left[1+\ln (1-x)\left(\frac{1}{x}-1\right)\right]+\frac{3}{8} x-\frac{3}{2} \operatorname{Li}_{2}(x),
$$

with $f_{R}^{\sigma}(0)=0$. At the fixed point $\tilde{g}^{*}=6 /(n+8) \epsilon+O\left(\epsilon^{2}\right)$, Eq. (88) can be cast in the expected scaling form (34) with

$$
\begin{aligned}
A_{R}^{\sigma} & =1+\tilde{g}^{*}\left[\frac{\pi^{2}}{4}-\frac{61+2 n}{24}\right]+O\left(\epsilon^{2}\right), \\
F_{R}^{\sigma}(x) & =1+\tilde{g}^{*} f_{R}^{\sigma}(x)+O\left(\epsilon^{2}\right) .
\end{aligned}
$$

\section{Longitudinal Connected Correlation function}

As in the case of $R_{\mathbf{q}}^{\sigma}$, the longitudinal correlation function gets a one-loop correction $C_{\mathbf{q}}^{\sigma, 0}(t, s)$ due the one-loop term in the magnetization $m(t)$ [see Eq. (85)]. This correction has been evaluated in Ref. [30], Eq. (B.29). In making use of that result we have to keep in mind that the factor $r_{d}$ in Ref. [30] accounts only for the contribution of longitudinal modes (i.e., $r_{d}$ would be $r_{\sigma}$ in the current notation) whereas here it contains also the contribution of transverse modes [see Eq. (83)]. The additional interaction vertices contribute via the Feynman diagrams depicted in Fig. 5, in terms of which

$$
C_{\mathbf{q}}^{\sigma}(t, s)=C_{\mathbf{q}}^{\sigma, 0}(t, s)+\frac{g_{0}}{2} I_{5}+g_{0} \frac{n-1}{18} I_{8}+g_{0} I_{6}+g_{0} \frac{n-1}{9} I_{9}-\frac{g_{0}}{2} I_{7}-g_{0} \frac{n-1}{6} I_{10}+O\left(g_{0}^{2}\right) .
$$

Using the results reported in Appendix B 2 we obtain the zero-momentum longitudinal correlation function $(x \equiv s / t)$

$C_{\mathbf{q}=0}^{\sigma}(t, s)=\frac{1}{2} s x^{3 / 2}\left[1+\tilde{g}\left(-\frac{n+8}{24} \ln x+\frac{37 \pi^{2}}{160}-\frac{25 n}{432}-\frac{18913}{8640}\right)+\tilde{g} f_{C}^{\sigma}(s / t)+O\left(\tilde{g}^{2}, \epsilon \tilde{g}\right)\right]$,

which has, at the RG fixed point, the expected scaling form with the correct exponents and

$$
\begin{aligned}
2 A_{C}^{\sigma} & =1+\tilde{g}^{*}\left(\frac{37 \pi^{2}}{160}-\frac{25 n}{432}-\frac{18913}{8640}\right)+O\left(\epsilon^{2}\right), \\
F_{C}^{\sigma}(x) & =1+\tilde{g}^{*} f_{C}^{\sigma}(x)+O\left(\epsilon^{2}\right),
\end{aligned}
$$

where $f_{C}^{\sigma}(x)$ is given by Eq. (B31). 


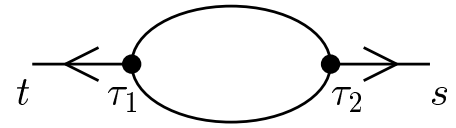

(5)
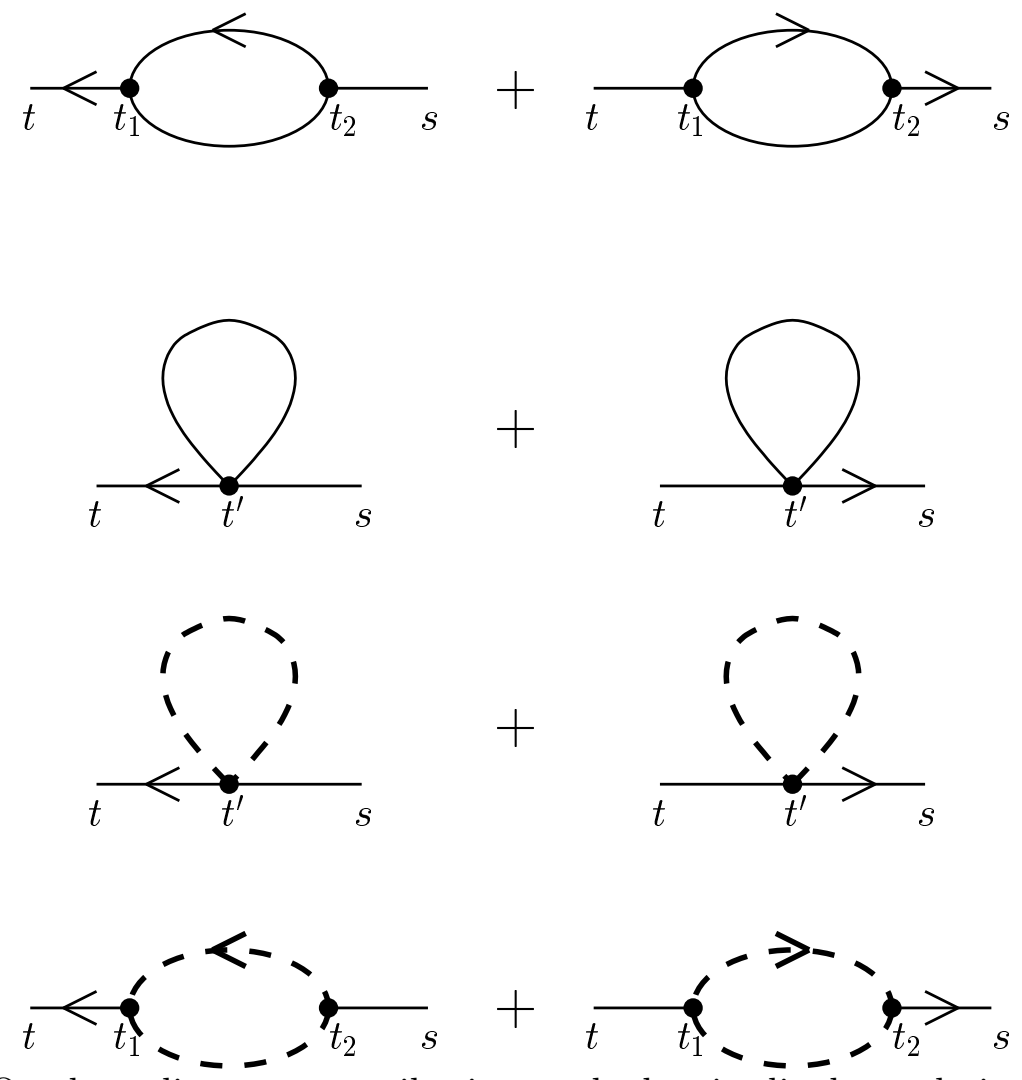

(6)

FIG. 5. One-loop diagrams contributing to the longitudinal correlation function $C_{\mathbf{q}}^{\sigma}(t, s)$. The corresponding expressions are indicated by $I_{i}(t, s), i=5, \ldots 10$ and are computed in Appendix B 2

\section{Longitudinal FDR}

It is now easy to compute the longitudinal FDR from Eqs. (40), (38), (90), and (94)

$$
X_{\sigma}^{\infty}=\frac{A_{R}^{\sigma}}{A_{C}^{\sigma}\left(1-\theta_{\sigma}\right)}=\frac{4}{5}-\frac{1895+76 n-162 \pi^{2}}{1800(8+n)} \epsilon+O\left(\epsilon^{2}\right),
$$

which for $n=1$ reproduces the result of Ref. [30] for the Ising model. Close to $d=4, X_{\sigma}^{\infty}$ $(n \geq 2)$ decreases as the dimensionality decreases. A monotonic behavior has to be expected down to the lower critical dimension $d=2$, where it is known that $X_{\sigma}^{\infty}=1 / 2$ [24]. In the limit $n \rightarrow \infty$ the FDR becomes

$$
X_{\sigma}^{\infty}(n=\infty)=\frac{4}{5}-\frac{19}{450} \epsilon+O\left(\epsilon^{2}\right),
$$



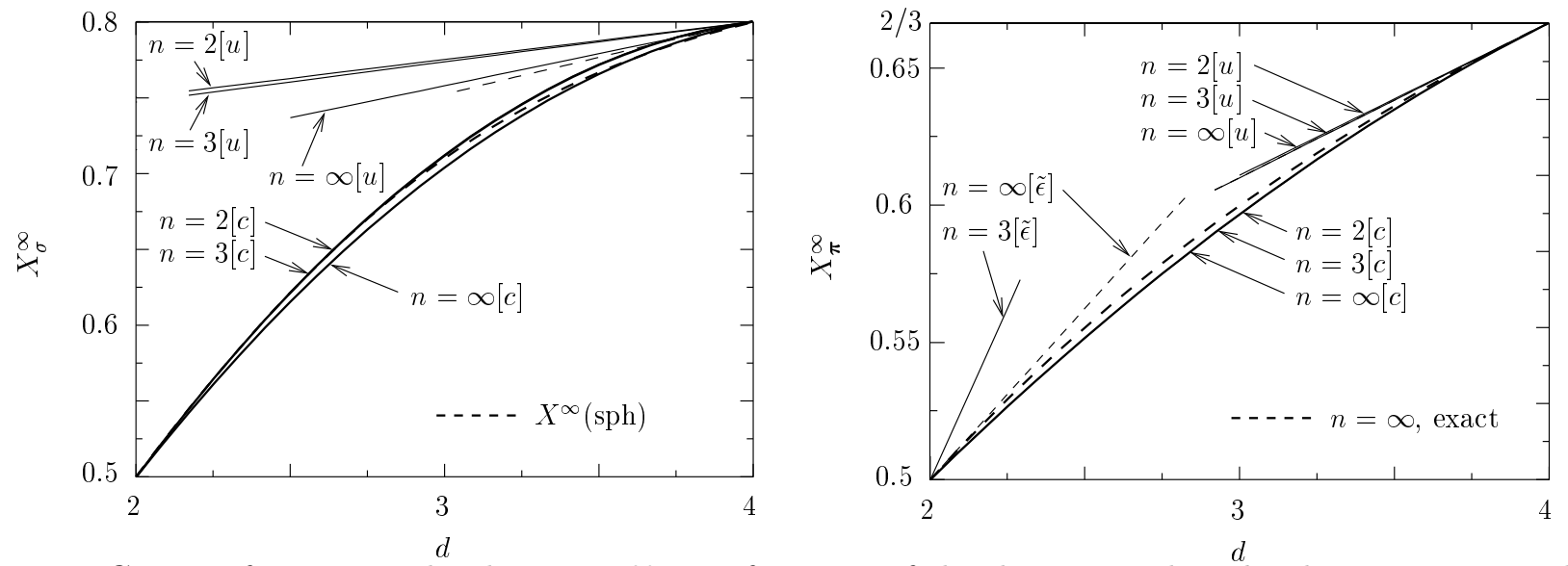

FIG. 6. Left: Longitudinal FDR $X_{\sigma}^{\infty}$ as a function of the dimensionality $d$. The unconstrained ([u], see Eq. (96)) and constrained ([c], see Eq. (101)) estimates for $n=2,3$ and $\infty$ are shown together with the result $X^{\infty}(\mathrm{sph})$ of Ref. [26] for the spherical model (dashed line). The dashed straight line indicates the expansion of $X^{\infty}$ (sph) close to $d=4$, which does not coincide with the limit $n \rightarrow \infty$ of Eq. (96). Right: Transverse FDR $X_{\boldsymbol{\pi}}^{\infty}$ as a function of the dimensionality $d$. The unconstrained ([u], see Eq. (115)) and constrained ([c], see Eq. (118)) estimates for $n=2,3$ and $\infty$ are shown together with the exact result (71) for $n=\infty$ (dashed line). For comparison we show also the results of the $\tilde{\epsilon}$-expansion $(\tilde{\epsilon}=d-2)$ of Ref. [24], Eq. (73), for $n=3$ and $n=\infty$.

which differs from the $\epsilon$-expansion of the result reported in Ref. [26] (see Eq. (8.116) therein) for the spherical model:

$$
X^{\infty}(\mathrm{sph})=\frac{4}{5}+\left(\frac{2 \ln 2}{15}-\frac{53}{900}-\frac{\pi^{2}}{120}\right) \epsilon+O\left(\epsilon^{2}\right) .
$$

For finite $n$, a first rough numerical estimate of $X_{\sigma}^{\infty}$ in the interesting and non-trivial physical dimension $d=3$ can be obtained by evaluating equation (96) for $\epsilon=1$. For instance we have $([u]$ denotes unconstrained estimates, to distinguish them from the constrained ones introduced below)

$$
\begin{aligned}
& X_{\sigma}^{\infty}[u](n=2)=0.775, \\
& X_{\sigma}^{\infty}[u](n=3)=0.774 .
\end{aligned}
$$

However, the reliability of such results can be questioned, both because they are based on a one-loop approximation and mainly because for $\epsilon=2$ one would find $X_{\sigma}^{\infty}[u] \sim 0.75$, which is quite far from the exact result $1 / 2$. To obtain more reliable estimates without computing the $O\left(\epsilon^{2}\right)$ contribution (which seems to be a difficult task, requiring the calculation of almost fifty Feynman diagrams) one can constrain $[c]$ the $O(\epsilon)$ result to assume the exact known value at $d=2$, as usually done for this kind of expressions (see, e.g., $[20,40]$ ). Assuming a smooth behavior in $\epsilon$ up to $\epsilon=2$ one gets

$$
X_{\sigma}^{\infty}[c]=\frac{1}{2}+\left(1-\frac{\epsilon}{2}\right)\left[\frac{3}{10}-\left(\frac{1895+76 n-162 \pi^{2}}{1800(8+n)}-\frac{3}{20}\right) \epsilon\right],
$$




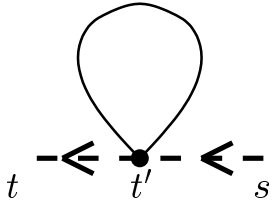

(11)

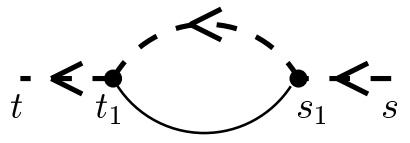

(13)

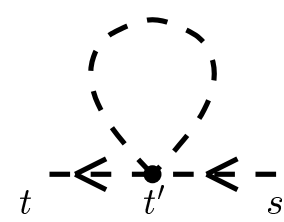

$(12)$

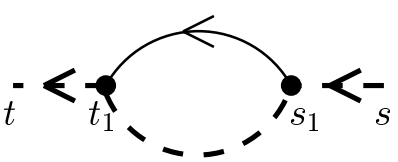

(14)

FIG. 7. One-loop diagrams contributing to the transverse response function $R_{\mathbf{q}}^{\boldsymbol{\pi}}(t, s)$. The corresponding expressions, indicated by $I_{i}(t, s), i=11, \ldots, 14$, are calculated in Appendix B 3 .

which has the same $\epsilon$-expansion as Eq. (96), assumes the exact value in $d=2$ and therefore it is expected to converge more rapidly to the correct three-dimensional result. The estimate (101) yields, for $\epsilon=1$,

$$
\begin{aligned}
& X_{\sigma}^{\infty}[c](n=2)=0.713, \\
& X_{\sigma}^{\infty}[c](n=3)=0.712,
\end{aligned}
$$

which are considerably smaller than the corresponding unconstrained estimates. A more robust field-theoretical estimate would require the knowledge of higher-order terms in the $\epsilon$-expansion. In Fig. 6 we compare the unconstrained $[u]$ and constrained $[c]$ estimates for $n=2,3, \infty$ as functions of the dimensionality $d$ ranging between the lower and the upper critical dimensions of the model (2 and 4, respectively). For comparison we report the exact result $X^{\infty}(\mathrm{sph})$ for the spherical model (dashed line), given by Eq. (8.113) in Ref. [26] (cp. also Fig. 4 therein). As already pointed out, our result (97) close to $d=4$ (see the straight line $n=\infty[u]$ in Fig. 6) differs from the corresponding one (98) obtained in Ref. [26] (represented as a straight dashed line in Fig. 6). On the other hand, the numerical discrepancy between these two expressions is quite small, resulting in a constrained estimate $X_{\sigma}^{\infty}[c](n=\infty)$ which differs from $X^{\infty}(\mathrm{sph})$ by at most $3 \%$.

\section{E. Transverse response function}

The transverse response function, like the longitudinal one, gets modified because of the one-loop contribution to $m(t)$ :

$$
\begin{aligned}
R_{\mathbf{q}=0}^{\pi, 0}(t, s) & =\mathrm{e}^{-q^{2}(t-s)-\frac{1}{3} \int_{s}^{t} \mathrm{~d} t^{\prime} m^{2}\left(t^{\prime}\right)} \\
& =\left(\frac{s}{t}\right)^{1 / 2}\left[1+\tilde{g}_{0} \frac{1}{1+\epsilon / 2} r_{d} \frac{t^{\epsilon / 2}-s^{\epsilon / 2}}{\epsilon / 2}+O\left(\tilde{g}_{0}^{2}\right)\right] \mathrm{e}^{-q^{2}(t-s)} .
\end{aligned}
$$


In addition, the interaction vertices in $\mathcal{L}_{1}$ [see Eq. (14)] yield the diagrammatic contributions depicted in Fig. 7, in terms of which the transverse response function reads

$$
R_{\mathbf{q}}^{\boldsymbol{\pi}}(t, s)=R_{\mathbf{q}}^{\boldsymbol{\pi}, 0}(t, s)-\frac{g_{0}}{6} I_{11}-g_{0} \frac{n+1}{6} I_{12}+\frac{g_{0}}{9} I_{13}+\frac{g_{0}}{9} I_{14}+O\left(g_{0}^{2}\right) .
$$

Using the expressions of $I_{i}, i=11, \ldots, 14$ reported in Appendix B 3 we find for $\mathbf{q}=0$ $(x \equiv s / t)$

$$
R_{\mathbf{q}=0}^{\boldsymbol{\pi}}(t, s)=x^{1 / 2}\left[1+\tilde{g}\left(-\frac{n+8}{24} \ln x-\frac{1}{24}+f_{R}^{\boldsymbol{\pi}}(x)+O\left(\tilde{g}^{2}, \epsilon \tilde{g}\right)\right)\right],
$$

where

$$
f_{R}^{\pi}(x)=\frac{\ln (1-x)+x}{4 x}-\frac{5}{24} x-\frac{1}{3} \ln (1-x)+\frac{1}{12} x \ln (1-x)
$$

$\left(f_{R}^{\boldsymbol{\pi}}(0)=0\right)$. Equation (106) displays, at the RG fixed point $\tilde{g}=\tilde{g}^{*}$, the correct scaling behavior [see Eq. (36)] with the proper exponents [see Eq. (76)] and

$$
\begin{aligned}
A_{R}^{\boldsymbol{\pi}} & =1-\frac{\tilde{g}^{*}}{24}+O\left(\epsilon^{2}\right), \\
F_{R}^{\boldsymbol{\pi}}(x) & =1+\tilde{g}^{*} f_{R}^{\boldsymbol{\pi}}(x)+O\left(\epsilon^{2}\right) .
\end{aligned}
$$

\section{F. Transverse correlation}

As in the case of $C_{\mathbf{q}}^{\sigma}$, the transverse correlation function gets a one-loop correction $C_{\mathbf{q}}^{\boldsymbol{\pi}, 0}(t, s)$ due the one-loop term in the magnetization $m(t)$ [see Eq. (85)]. This correction can be computed from Eqs. (24) and (104), finding

$$
\begin{aligned}
C_{\mathbf{q}=0}^{\boldsymbol{\pi}}(t, s) & =2 \int_{0}^{s} \mathrm{~d} t^{\prime} R_{\mathbf{q}=0}^{\boldsymbol{\pi}, 0}\left(t, t^{\prime}\right) R_{\mathbf{q}=0}^{\boldsymbol{\pi}, 0}\left(s, t^{\prime}\right) \\
& =s x^{1 / 2}\left\{1+\tilde{g}_{0} \frac{r_{d}}{1+\epsilon / 2} s^{\epsilon / 2}\left[\frac{x^{-\epsilon / 2}-1}{\epsilon / 2}+\frac{2}{2+\epsilon / 2}\right]\right\}+O\left(\tilde{g}_{0}^{2}\right) .
\end{aligned}
$$

The additional vertices contribute via the Feynman diagrams depicted in Fig. 8, according to

$$
C_{\mathbf{q}}^{\boldsymbol{\pi}}(t, s)=C_{\mathbf{q}}^{\boldsymbol{\pi}, 0}(t, s)-g_{0} \frac{n+1}{6} I_{15}-\frac{g_{0}}{6} I_{16}+\frac{g_{0}}{9} I_{17}+\frac{g_{0}}{9} I_{18}+\frac{g_{0}}{9} I_{19}+O\left(g_{0}^{2}\right) .
$$

Using the results reported in Appendix B 4 for $I_{i}, i=15, \ldots, 19$, we obtain the zeromomentum transverse correlation function

$$
C^{\boldsymbol{\pi}}(t, s)_{\mathbf{q}=0}=s\left(\frac{s}{t}\right)^{1 / 2}\left[1+\tilde{g}\left(-\frac{n+8}{24} \ln x+\frac{127}{432}+\frac{n}{24}+f_{C}^{\boldsymbol{\pi}}(s / t)\right)+O\left(\tilde{g}^{2}, \epsilon \tilde{g}\right)\right],
$$

which displays the expected scaling behavior [cp. Eq. (37)] with the proper exponents [see Eq. (76)] and

$$
\begin{aligned}
A_{C}^{\boldsymbol{\pi}} & =1+\tilde{g}^{*}\left(\frac{127}{432}+\frac{n}{24}\right)+O\left(\epsilon^{2}\right), \\
F_{C}^{\boldsymbol{\pi}}(x) & =1+\tilde{g}^{*} f_{C}^{\boldsymbol{\pi}}(x)+O\left(\epsilon^{2}\right),
\end{aligned}
$$

where $f_{C}^{\boldsymbol{\pi}}(x)$ is given by Eq. (B53). 

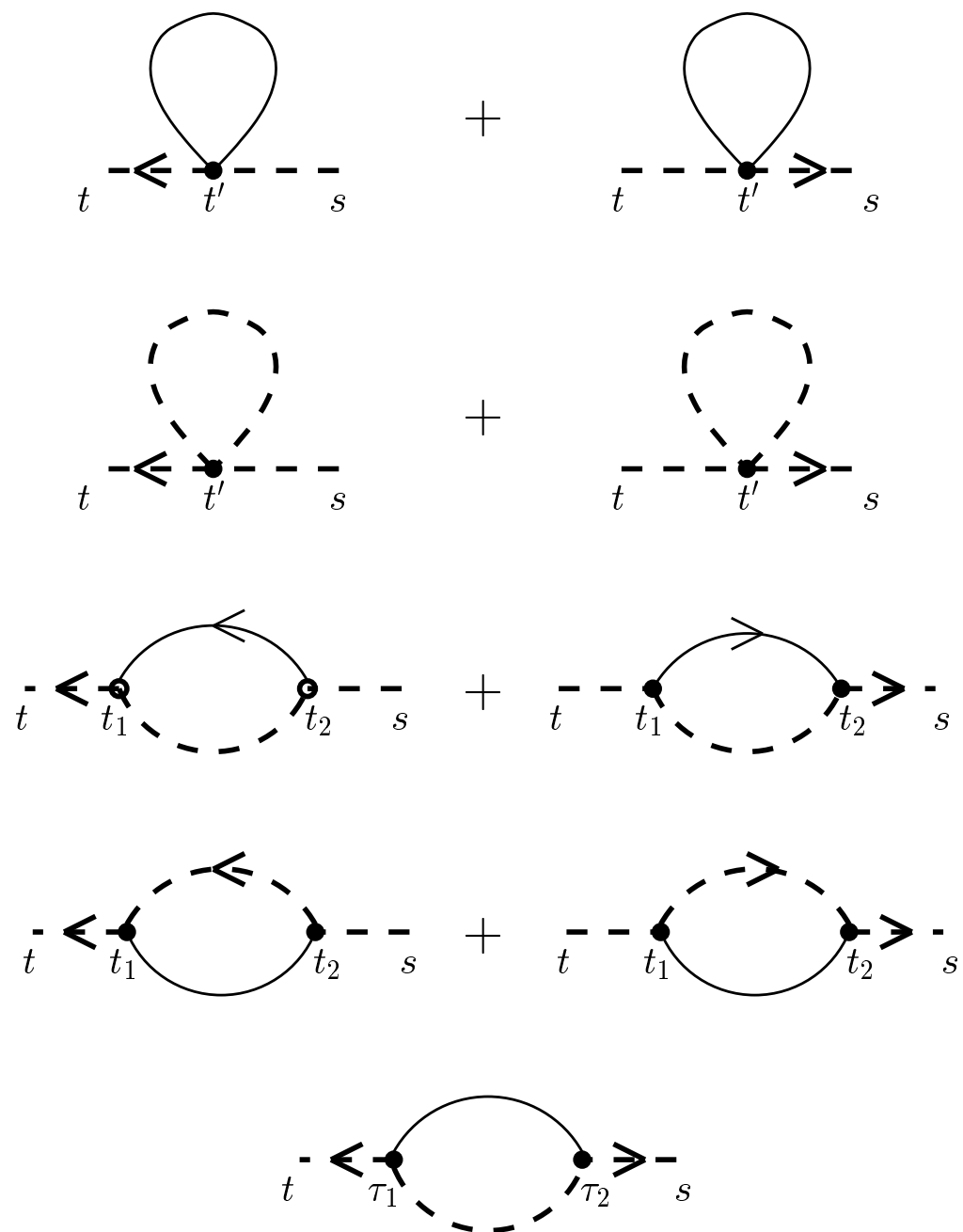

FIG. 8. One-loop diagrams contributing to the transverse correlation function $C_{\mathbf{q}}^{\boldsymbol{\pi}}(t, s)$. The corresponding expressions, indicated by $I_{i}(t, s), i=15, \ldots, 19$, are reported in Appendix B 4 .

\section{G. The transverse FDR}

The expressions of the transverse response and correlation functions allow the determination of the FDR according to Eq. (40) [see also Eqs. (108), (113), and (39)]

$$
X_{\boldsymbol{\pi}}^{\infty}=\frac{A_{R}^{\pi}}{A_{C}^{\boldsymbol{\pi}}\left(1-\theta_{\boldsymbol{\pi}}\right)}=\frac{2}{3}-\frac{49+6 n}{108(8+n)} \epsilon+O\left(\epsilon^{2}\right),
$$

which is a decreasing function of $\epsilon$, a feature that we expect to persist down to the lower critical dimension $(d=2$ for the case of interest $n \geq 2)$. Note that the coefficient of the correction of $O(\epsilon)$, i.e., $1 / 18+1 /[108(8+n)]$, changes less than $2 \%$ with $n \geq 2$ and therefore $X_{\boldsymbol{\pi}}^{\infty}$, at least close to $d=4$, is practically independent of the actual number $n-1$ of transverse components. In the limit $n \rightarrow \infty, X_{\boldsymbol{\pi}}^{\infty}$ reduces to

$$
X_{\pi}^{\infty}(n=\infty)=\frac{2}{3}-\frac{1}{18} \epsilon+O\left(\epsilon^{2}\right),
$$


in agreement with the $\epsilon$-expansion of the exact result $X_{\boldsymbol{\pi}}^{\infty}(n=\infty)=d /(d+2)$, obtained in Sec. IV. As for finite $n$, we proceed as in the case of the longitudinal FDR, first by giving unconstrained estimates (obtained by setting $\epsilon$ to the proper value in Eq. (115) and neglecting $O\left(\epsilon^{2}\right)$ corrections) and then improving them by taking advantage of the known value for $d=2$. The direct estimates for the physical dimension $d=3$ are (they differ on the fourth digit)

$$
X_{\boldsymbol{\pi}}^{\infty}[u](n=2,3)=0.610 .
$$

These unconstrained $[u]$ estimates are expected to work better than in the longitudinal case, because for $\epsilon=2$ (i.e., $d=2)$ they give $X_{\boldsymbol{\pi}}^{\infty}[u](n=2,3) \simeq 0.55$, which is only $10 \%$ above the exact value $1 / 2$. To confirm this expectation we compute the constrained FDR

$$
X_{\boldsymbol{\pi}}^{\infty}[c]=\frac{1}{2}+\left(1-\frac{\epsilon}{2}\right)\left[\frac{1}{6}-\left(\frac{49+6 n}{108(8+n)}-\frac{1}{12}\right) \epsilon\right],
$$

which gives, in three dimensions,

$$
X_{\boldsymbol{\pi}}^{\infty}[c](n=2,3)=0.597 .
$$

This estimate is indeed quite close to the unconstrained one, suggesting that in this case the one-loop expression provides more reliable results than it does for the longitudinal FDR. In Fig. 6 (right panel) a comparison between the various estimates as functions of the dimensionality $d$ is provided for $2 \leq d \leq 4$. The unconstrained ones for $n=2,3$, and $\infty$ (denoted by $n=2[u], n=3[u]$, and $n=\infty[u]$, respectively) almost coincide due to the weak dependence on $n$ of the expression (115). As a consequence, the corresponding constrained estimates ( $n=2[c], n=3[c]$, and $n=\infty[c]$, respectively) are not distinguishable on this scale. For comparison we report (dashed line) the exact prediction in the limit $n \rightarrow \infty$ (see Eq. (71)) and the prediction (73) for the $\tilde{\epsilon}$-expansion $(\tilde{\epsilon}=d-2)$ close to the lower critical dimension for $n=3$ and $n=\infty$ (denoted by $n=3[\tilde{\epsilon}]$ and $n=\infty[\tilde{\epsilon}]$, respectively). As already pointed out in Sec. IV the result $n=\infty[\tilde{\epsilon}]$ is indeed tangential to the exact result $n=\infty$, exact (dashed line, Eq. (71)). The constrained estimate $n=3[c]$ provided by Eq. (118) does not account completely for the behavior of $X_{\sigma}^{\infty}(n=\infty)$ close to $d=2$, as predicted by Eq. (73), but reproduces only its value for $d=2$. Reliable interpolation formulas accounting properly for both the behaviors close to $d=2$ and $d=4$ require the knowledge of higher order terms in $\tilde{\epsilon}$ and $\epsilon$ than those currently available.

\section{CONCLUSIONS}

In this paper we have studied the non-equilibrium dynamics of a system belonging to the $O(n)$ universality class and relaxing at criticality according to a purely dissipative dynamics from a state with initial magnetization $M_{0}$ (induced via an external magnetic field). In the

aging regime $\left(t \gg s \gg \tau_{m} \sim M_{0}^{-1 / \kappa}\right.$ ) we found that the zero-momentum transverse $(\boldsymbol{\pi})$ and longitudinal $(\sigma)$ response and connected correlation functions have the scaling forms 


$$
\begin{aligned}
& R_{\mathbf{q}=0}^{\sigma}(t, s)=A_{R}^{\sigma}(t-s)^{a}(t / s)^{\theta_{\sigma}} F_{R}^{\sigma}(s / t), \\
& C_{\mathbf{q}=0}^{\sigma}(t, s)=A_{C}^{\sigma} s(t-s)^{a}(t / s)^{\theta_{\sigma}} F_{C}^{\sigma}(s / t), \\
& R_{\mathbf{q}=0}^{\boldsymbol{\pi}}(t, s)=A_{R}^{\boldsymbol{\pi}}(t-s)^{a}(t / s)^{\theta_{\pi}} F_{R}^{\pi}(s / t), \\
& C_{\mathbf{q}=0}^{\boldsymbol{\pi}}(t, s)=A_{C}^{\pi} s(t-s)^{a}(t / s)^{\theta_{\pi}^{\prime}} F_{C}^{\pi}(s / t),
\end{aligned}
$$

independently of the actual value of $M_{0} \neq 0$. We argued that the exponents $\theta_{\sigma, \boldsymbol{\pi}}$ and $\theta_{\sigma, \boldsymbol{\pi}}^{\prime}$ are not new non-equilibrium quantities, but they can be written in terms of equilibrium exponents as

$$
\begin{aligned}
& \theta_{\sigma}=\theta_{\sigma}^{\prime}=-\frac{\beta \delta}{\nu z}, \\
& \theta_{\boldsymbol{\pi}}=\theta_{\boldsymbol{\pi}}^{\prime}=-\frac{\beta}{\nu z} .
\end{aligned}
$$

The two response and correlation functions define two (in principle different) FDRs $X_{\sigma}^{\infty}$ and $X_{\pi}^{\infty}$.

We solved exactly the dynamics of the transverse modes within the Gaussian approximation and we derived all the relevant universal ratios and scaling functions (the dynamics of the longitudinal modes was already discussed in Ref. [30]). In particular we found that the FDRs of longitudinal and transverse modes are different, making the effective temperature (defined via $X^{\infty}$ ) observable-dependent already within the Gaussian approximation. Then we solved exactly the dynamics of the transverse modes in the limit $n \rightarrow \infty$. The resulting FDR interpolates correctly (as a function of the aging time) from the well-known spherical model value $X^{\infty}=1-2 / d[8]$ at $M_{0}=0$ and the new result

$$
X_{\boldsymbol{\pi}}^{\infty}=\frac{d}{d+2},
$$

at any $M_{0} \neq 0$. This result coincides with the one for local spin observables in the spherical model [26].

In order to provide more accurate predictions in physical dimensions $d=2,3$ for the physical models with $n=2,3$ we computed the first-order correction in the $\epsilon$-expansion to the equation of motion, to the response and correlation functions and from them we obtained the FDRs. In particular for the longitudinal modes we found

$$
X_{\sigma}^{\infty}=\frac{4}{5}-\frac{1895+76 n-162 \pi^{2}}{1800(8+n)} \epsilon+O\left(\epsilon^{2}\right),
$$

which differs for $n=\infty$ from the $\epsilon$-expansion of the corresponding spherical model result [26]. To our knowledge this is the first example of a universal quantity taking different values in these two models, which are known to be equivalent in equilibrium [41]. It is not yet clear to us whether this discrepancy is actually due to an effectively different initial state or to nonGaussian corrections to the action which have to be taken into account for the longitudinal mode (see Ref. [26]). For comparison, it would be interesting to calculate the leading nonGaussian contributions to the $O(\infty)$ model. For finite $n$ we provided an estimate of $X_{\sigma}^{\infty}$ by 
constraining it to assume the known value in two dimensions (see Eq. (101)), resulting in $X_{\sigma}^{\infty}(n=2,3) \simeq 0.71$ in three dimensions. For the transverse FDR we obtained

$$
X_{\boldsymbol{\pi}}^{\infty}=\frac{2}{3}-\frac{49+6 n}{108(8+n)} \epsilon+O\left(\epsilon^{2}\right) .
$$

The constrained estimates (see Eq. (118)) in $d=3$ yield $X_{\boldsymbol{\pi}}^{\infty}(n=2,3) \simeq 0.60$.

An interesting extension of the work presented here would be to consider the nonequilibrium evolution at criticality of the $O(n)$ model starting from an equilibrium magnetized state which has been prepared in the low-temperature phase $T<T_{c}$ in zero magnetic field. In this case the transverse modes are critical even in the initial state, affecting in a non-trivial way the ensuing evolution.

\section{ACKNOWLEDGMENTS}

We thank P. Sollich for very useful discussions. PC acknowledges the financial support from the Stichting voor Fundamenteel Onderzoek der Materie (FOM).

\section{APPENDIX A: DETERMINATION OF THE $h$ FUNCTION}

In this appendix we determine the function $h(x)$ [introduced in Eq. (60)] which characterizes completely the dynamics of the transverse modes in the limit $n \rightarrow \infty$.

First of all we express the self-consistency condition Eq. (57) in terms of $h$ only. To this end we note that Eq. (60) implies:

$$
\hat{r}(t)=\Lambda^{2} \frac{h^{\prime}\left(2 \Lambda^{2} t\right)}{h\left(2 \Lambda^{2} t\right)}
$$

and that, from Eqs. (62) and (56),

$$
\int^{\Lambda}(\mathrm{d} q) C_{\mathbf{q}}^{\boldsymbol{\pi}}(t, t)=2 \int_{0}^{t} \mathrm{~d} t^{\prime} \frac{h\left(2 \Lambda^{2} t^{\prime}\right)}{h\left(2 \Lambda^{2} t\right)} \int^{\Lambda}(\mathrm{d} q) \mathrm{e}^{-2 \mathbf{q}^{2}\left(t-t^{\prime}\right)}=2 N_{d} \Lambda^{d} \int_{0}^{t} \mathrm{~d} t^{\prime} \frac{h\left(2 \Lambda^{2} t^{\prime}\right)}{h\left(2 \Lambda^{2} t\right)} K_{d}\left(2 \Lambda^{2}\left(t-t^{\prime}\right)\right),
$$

where we have introduced the kernel

$$
K_{d}(\tau) \equiv N_{d}^{-1} \Lambda^{-d} \int^{\Lambda}(\mathrm{d} q) \mathrm{e}^{-\mathbf{q}^{2} \tau / \Lambda^{2}}
$$

Its explicit expression depends on the specific implementation of the large-momentum cut off. For later convenience we have introduced the factor $N_{d}=2 /\left[(4 \pi)^{d / 2} \Gamma(d / 2)\right]$ in the definition of $K_{d}$. Then we compute

$$
\int^{\Lambda}(\mathrm{d} q) C_{\mathbf{q}}^{\boldsymbol{\pi}}(\infty, \infty)=\int^{\Lambda}(\mathrm{d} q) \frac{1}{\mathbf{q}^{2}+\hat{r}_{\infty}}=N_{d} \Lambda^{d-2} I_{d}\left(\hat{r}_{\infty} / \Lambda^{2}\right),
$$


where $I_{d}(z)$ is given by

$$
I_{d}(z) \equiv N_{d}^{-1} \Lambda^{-(d-2)} \int^{\Lambda}(\mathrm{d} q) \frac{1}{\mathbf{q}^{2}+\Lambda^{2} z}
$$

$\left(I_{d}(0)\right.$ is finite for $\left.d>2\right)$. Using these results and Eq. (61), the consistency condition (57) can be expressed in terms of $h$ only

$$
\Lambda^{2} \frac{h^{\prime}(\tau)}{h(\tau)}=\hat{r}_{\infty}+\frac{g N_{d}}{6} \Lambda^{d-2}\left\{\int_{0}^{\tau} \mathrm{d} \tau^{\prime} \frac{h\left(\tau^{\prime}\right)}{h(\tau)} K_{d}\left(\tau-\tau^{\prime}\right)-I_{d}\left(\hat{r}_{\infty} / \Lambda^{2}\right)\right\}+\frac{m_{0}^{2}}{6} \frac{1}{h(\tau)}
$$

where $\tau=2 \Lambda^{2} t$ and $\tau^{\prime}=2 \Lambda^{2} t^{\prime}$ are dimensionless time variables. This equation turns into a linear differential equation for $h$ :

$$
h^{\prime}(\tau)=\frac{\hat{r}_{\infty}}{\Lambda^{2}} h(\tau)+u\left\{\int_{0}^{\tau} \mathrm{d} \tau^{\prime} h\left(\tau^{\prime}\right) K_{d}\left(\tau-\tau^{\prime}\right)-I_{d}\left(\hat{r}_{\infty} / \Lambda^{2}\right) h(\tau)\right\}+\frac{1}{6} \frac{m_{0}^{2}}{\Lambda^{2}},
$$

where the dimensionless coupling constant is given by $u \equiv g N_{d} \Lambda^{d-4} / 6$ and which can be easily solved by using the Laplace transform $\hat{g}(s)=\mathcal{L}[g(\tau)] \equiv \int_{0}^{\infty} \mathrm{d} \tau g(\tau) \mathrm{e}^{-s \tau}$, defined in the half-plane $\operatorname{Re}(s)>\gamma$, where $\gamma$ depends on the analytical properties of $g$. Once $\hat{g}(s)$ is known, the original function is obtained via the inverse transform $g(\tau)=(2 \pi i)^{-1} \int_{\gamma^{\prime}-i \infty}^{\gamma^{\prime}+i \infty} \mathrm{d} s \hat{g}(s) \mathrm{e}^{s \tau}$ for $\gamma^{\prime}>\gamma$. Transforming Eq. (A7) one gets $\mathcal{L}\left[h^{\prime}(\tau)\right]=-h(0)+s \hat{h}(s)$ where, from the definition $(60), h(0)=1 / 2$, and

$$
\int_{0}^{\infty} \mathrm{d} \tau \mathrm{e}^{-s \tau} \int_{0}^{\tau} \mathrm{d} \tau^{\prime} h\left(\tau^{\prime}\right) K_{d}\left(\tau-\tau^{\prime}\right)=\int_{0}^{\infty} \mathrm{d} \tau^{\prime} \mathrm{e}^{-s \tau^{\prime}} h\left(\tau^{\prime}\right) \int_{\tau^{\prime}}^{\infty} \mathrm{d} \tau \mathrm{e}^{-s\left(\tau-\tau^{\prime}\right)} K_{d}\left(\tau-\tau^{\prime}\right)=\hat{h}(s) \hat{K}_{d}(s),
$$

where (see Eq. (A3))

$$
\hat{K}_{d}(s) \equiv \int_{0}^{\infty} \mathrm{d} \tau \mathrm{e}^{-s \tau} K_{d}(\tau)=\Lambda^{-d} \int_{0}^{\infty} \mathrm{d} \tau \mathrm{e}^{-s \tau} \int^{\Lambda}(\mathrm{d} q) \mathrm{e}^{-\mathbf{q}^{2} \tau / \Lambda^{2}}=\Lambda^{-d} \int^{\Lambda}(\mathrm{d} q) \frac{1}{\mathbf{q}^{2} / \Lambda^{2}+s}=I_{d}(s) .
$$

Accordingly, Eq. (A7) becomes

$$
-\frac{1}{2}+s \hat{h}(s)=\frac{\hat{r}_{\infty}}{\Lambda^{2}} \hat{h}(s)+u\left[I_{d}(s)-I_{d}\left(\hat{r}_{\infty} / \Lambda^{2}\right)\right] \hat{h}(s)+\frac{1}{6} \frac{m_{0}^{2}}{\Lambda^{2}} \frac{1}{s},
$$

whose solution is

$$
\hat{h}(s)=\frac{\frac{1}{2}+\frac{1}{6} \frac{m_{0}^{2}}{\Lambda^{2}} \frac{1}{s}}{s-\frac{\hat{r}_{\infty}}{\Lambda^{2}}-u\left[I_{d}(s)-I_{d}\left(\hat{r}_{\infty} / \Lambda^{2}\right)\right]} .
$$

First we note that, as expected, $\hat{h}(s)$ has a pole for $s=s_{\infty} \equiv \hat{r}_{\infty} / \Lambda^{2}$ independently of the value of $u$. For $u=0, \hat{h}(s)$ is analytic in the complex plane except for the poles at $s=s_{\infty}$ and $s=0$. Accordingly, the corresponding inverse Laplace transform is

$$
h(\tau)=\frac{1}{2} \mathrm{e}^{s_{\infty} \tau}+\frac{1}{6} \frac{m_{0}^{2}}{\Lambda^{2}} \frac{1}{s_{\infty}}\left(\mathrm{e}^{s_{\infty} \tau}-1\right)
$$




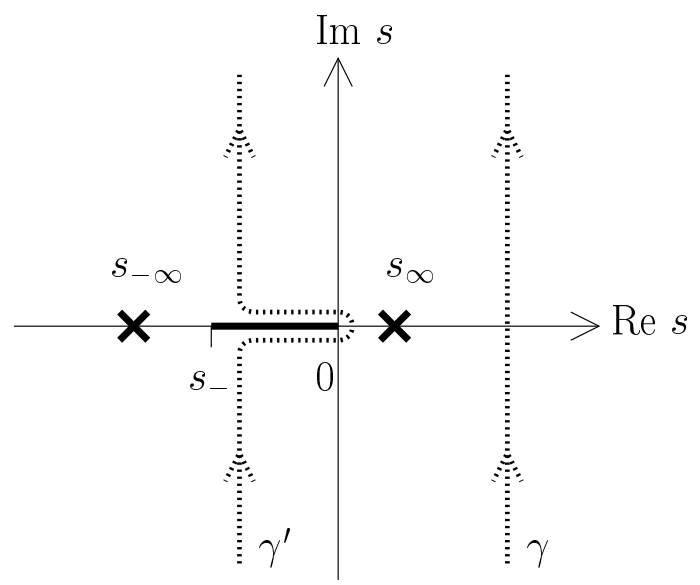

FIG. 9. Analytic structure of $\hat{h}(s)$ and contour of integration for the computation of its inverse Laplace transform. Poles are indicated by a cross, whereas the branch cut by the thick solid line. $\gamma$ is the original contour of integration to compute the inverse transform of $\hat{h}(s)$, whereas $\gamma^{\prime}$ is its convenient deformation in the case $s_{\infty}=0$ (see Appendix A 1).

i.e.,

$$
h\left(2 \Lambda^{2} t\right)=\frac{1}{2} \mathrm{e}^{2 \hat{r}_{\infty} t}+\frac{1}{6} \frac{m_{0}^{2}}{\hat{r}_{\infty}}\left(\mathrm{e}^{2 \hat{r}_{\infty} t}-1\right),
$$

which is actually independent of $\Lambda$, as expected. From this expression one recovers all the Gaussian results of Sec. III in the limit $\hat{r}_{\infty} \rightarrow 0$ (corresponding to the critical point).

Because of $I_{d}(s), \hat{h}(s)$ has a branch cut on the real axis for $s_{-}<\operatorname{Re}(s)<0$ and $u>0$, in addition to the poles for $s=s_{\infty}$ and $s=0$. If $s_{-}$is finite then an isolated pole $s_{-\infty}$ is also present on the real axis with $\operatorname{Re}\left(s_{-\infty}\right)<s_{-}$(see Appendix A 1). In the scaling limit we are interested in, $\Lambda^{2} t \gg 1(\tau \gg 1)$, the relevant contribution in the inverse Laplace transform comes from the singularities with the largest real part. If $\hat{r}_{\infty}>0$, this is given by $s=s_{\infty}$, leading, as in the Gaussian case, to an expected exponential decay of $h^{-1}(\tau)$. For $\hat{r}_{\infty}=0$, instead,

$$
\hat{h}(s)=\frac{\frac{1}{2}+\frac{1}{6} \frac{m_{0}^{2}}{\Lambda^{2}} \frac{1}{s}}{s-u\left[I_{d}(s)-I_{d}(0)\right]},
$$

and the leading singularity comes from the branch cut. Using the asymptotic expansion for $I_{d}(s)$ derived in Appendix A 2 we can write the denominator of Eq. (A14) as

$$
s-u\left[I_{d}(s)-I_{d}(0)\right]=a_{0} s^{d / 2-1}+b_{0} s+\sum_{k=1}^{\infty}\left[a_{k} s^{d / 2-1+k}+b_{k} s^{1+k}\right]
$$

where

$$
a_{0}=u \kappa_{d} \quad \text { and } \quad b_{0}=1-u \alpha_{d}
$$

and $\kappa_{d}=\pi /[2 \sin (\pi(d-2) / 2)]$ is a universal coefficient, i.e., independent of the actual regularization procedure, whereas $\alpha_{d}$ does depend on it (with the universal feature $\alpha_{4-\epsilon} \sim$ 
$1 / \epsilon$ for $\epsilon \rightarrow 0$ ). Accordingly, for $s \rightarrow 0$ and $2<d<4$ (so that $\epsilon=4-d>0$ ) one has

$$
\frac{1}{s-u\left[I_{d}(s)-I_{d}(0)\right]}=a_{0}^{-1} s^{-1+\epsilon / 2}\left[1+\sum_{m, n=0}^{\infty} c_{m, n} s^{m+n \epsilon / 2}\right],
$$

where $c_{0,0}=0, c_{0, n}=\left(-b_{0} / a_{0}\right)^{n}$ and all the other coefficients can be computed in terms of $a_{k}$ and $b_{k}$. [Note that for $d>4$ the leading behavior for $s \rightarrow 0$ is, apart from a coefficient, the same as in the case $u=0$, leading to the expected Gaussian-like behavior.] The inverse Laplace transform of $\hat{h}(s)$ for $\tau \gg 1$ is dominated by the behavior of $\hat{h}(s)$ for $s \rightarrow 0$, and it can be computed following a suitable contour of integration in the complex plane (indicated as $\gamma^{\prime}$ in Fig. 9) on the two sides of the branch cut. Recalling that $\mathcal{L}^{-1}\left[1 / s^{\alpha}\right]=\tau^{\alpha-1} / \Gamma(\alpha)$, one concludes that

$$
\begin{aligned}
h(\tau \gg 1) & =\frac{a_{0}^{-1}}{2}\left[\frac{\tau^{-\epsilon / 2}}{\Gamma(1-\epsilon / 2)}+\sum_{n=1}^{\left\lfloor\frac{2}{\epsilon}\right\rfloor-1} \frac{c_{0, n}}{\Gamma(1-(n+1) / 2 \epsilon)} \tau^{-(n+1) / 2 \epsilon}\right] \\
& +\frac{a_{0}^{-1}}{6} \frac{m_{0}^{2}}{\Lambda^{2}}\left[\frac{\tau^{1-\epsilon / 2}}{\Gamma(2-\epsilon / 2)}+\sum_{n=1}^{\left\lfloor\frac{4}{\epsilon}\right\rfloor-1} \frac{c_{0, n} \tau^{1-(n+1) / 2 \epsilon}}{\Gamma(2-(n+1) / 2 \epsilon)}+\sum_{n=1}^{\left\lfloor\frac{2}{\epsilon}\right\rfloor-1} \frac{c_{1, n} \tau^{-(n+1) / 2 \epsilon}}{\Gamma(1-(n+1) / 2 \epsilon)}\right] \\
& +O\left(\tau^{-1}\right) .
\end{aligned}
$$

The confluent corrections $\sim \tau^{-n \epsilon / 2}(n>2)$ to the leading scaling behavior vanish identically if $c_{0, n}=0$, i.e., for $b_{0}=0$. According to Eq. (A16) this occurs if $u=u^{*}=\alpha_{d}^{-1}$, which is indeed the fixed-point value for $u$ (note that if dimensional regularization is employed, this leads to the well-known result $\left.u^{*}=\epsilon\right)$.

Accordingly, the scaling limit (i.e., $\Lambda \rightarrow \infty$ with $m_{0}^{2} t$ fixed) for $2<d<4$ is characterized by the fixed-point expression

$$
h\left(2 \Lambda^{2} t \gg 1\right)=\frac{1}{u^{*}} \frac{\kappa_{d}^{-1}}{2 \Gamma((d-2) / 2)}\left(2 \Lambda^{2} t\right)^{-\epsilon / 2}\left[1+\frac{4}{3(d-2)} m_{0}^{2} t+O\left(\left(2 \Lambda^{2} t\right)^{-\epsilon / 2}\right)\right],
$$

which is the result reported in Eq. (63).

\section{Poles of $\hat{h}(s)$}

Here we investigate the properties of analyticity for $s \in \mathbb{C}$ of

$$
\hat{h}(s)=\frac{\frac{1}{2}+\frac{1}{6} \frac{m_{0}^{2}}{\Lambda^{2}} \frac{1}{s}}{s-\rho-u\left[I_{d}(s)-I_{d}(\rho)\right]},
$$

where $\rho \equiv \hat{r}_{\infty} / \Lambda^{2}>0$. Clearly, the numerator has a pole for $s=0$ whereas the denominator vanishes for $s=\rho$, independently of $u>0 . I_{d}(s)$ [see Eq. (A5)] has a branch cut on the real axis for $s_{-} \equiv-\mathbf{q}_{\max }^{2} / \Lambda^{2}<\operatorname{Re} s<0$, where $\mathbf{q}_{\max }$ is the maximum momentum in the integration domain $\left(\left|\mathbf{q}_{\max }\right| \sim \Lambda\right)$. Depending on the actual definition of the momentum 
integral $\int^{\Lambda}(\mathrm{d} q), s_{-}$might be not finite. Possible additional poles are given by the solution of

$$
s-\rho-u\left[I_{d}(s)-I_{d}(\rho)\right]=0 .
$$

Note that, as a consequence of Eq. (A5),

$$
I_{d}(s)-I_{d}(\rho)=-(s-\rho) N_{d}^{-1} \Lambda^{4-d} \int^{\Lambda}(\mathrm{d} q) \frac{1}{\left(\mathbf{q}^{2}+\Lambda^{2} s\right)\left(\mathbf{q}^{2}+\Lambda^{2} \rho\right)},
$$

and therefore additional poles are given by the solutions of

$$
J_{d}(s) \equiv N_{d}^{-1} \Lambda^{4-d} \int^{\Lambda}(\mathrm{d} q) \frac{1}{\left(\mathbf{q}^{2}+\Lambda^{2} s\right)\left(\mathbf{q}^{2}+\Lambda^{2} \rho\right)}=-\frac{1}{u} .
$$

It is easy to see that the imaginary part of the integral vanishes only on the real axis and therefore possible poles have to be located there and we can restrict our search to $s \in\left(-\infty, s_{-}\right)$given that $J_{d}\left(s \in \mathbb{R}^{+}\right)>0$. Note that $J_{d}\left(s<s_{-}\right) \leq 0, J_{d}(s \rightarrow-\infty)=0^{-}$, and that $J_{d}^{\prime}(s)<0$ (as it is clear by differentiating the integral). In addition, $J_{d}\left(s \rightarrow s_{-}\right)$diverges logarithmically and therefore Eq. (A23) admits a unique solution $s_{-\infty}<s_{-}$for $u \neq 0$. In the case of a regularization with a sharp cut-off $\Lambda$ one finds $s_{-}=-1$ and it is easy to determine the asymptotic behavior of $J_{d}(s)$ for $s \rightarrow-1^{-}: J_{d}\left(s \rightarrow-1^{-}\right)=-(1+\rho)^{-1} \ln (-1-s)+$ finite and therefore Eq. (A23) admits a unique solution $s_{-\infty}<-1$ for $u \neq 0$. In particular, for $u$ small one has $s_{-\infty} \simeq-1-\mathrm{e}^{-2(1+\rho) / u}$.

\section{Expansion of $I_{d}(s)$}

Let us consider a possible definition of the regularized integral, via a cut-off function $\varphi(x)$ (defined for $x \in \mathbb{R}^{+}$), i.e.,

$$
\int^{\Lambda}(\mathrm{d} q) \mapsto \int_{\mathbb{R}^{d}}(\mathrm{~d} q) \varphi\left(\mathbf{q}^{2} / \Lambda^{2}\right)
$$

where we assume that $\varphi$ is a smooth function which does not change the small-momentum behavior of the integrand, i.e., $\varphi(0)=1$ and decays exponentially fast for $x \rightarrow \infty$. For later convenience we also assume that $\varphi(x)$ can be expanded around $x=0$ (or, at least that $\varphi^{\prime}(0)$ is finite). These properties are also encoded in the analytic properties of the Mellin transform $\bar{\varphi}(s)$ of $\varphi(x)$, defined as

$$
\bar{\varphi}(z) \equiv \int_{0}^{\infty} \mathrm{d} x x^{z-1} \varphi(x)
$$

which converges in the complex plane for $\operatorname{Re}(z)>0$ (in case of an algebraic decay of $\varphi(x)$ for $x \rightarrow \infty$ this domain becomes a strip). Its analytic extension to $z \in \mathbb{C}$ is characterized by poles on the real axis for the non-positive integers $n=0,-1,-2, \ldots$ with residues $\varphi^{(n)}(0) / n$ !, due to the small- $x$ behavior of $\varphi(x)$. The original function $\varphi(x)$ is recovered by computing

$$
\varphi(x)=\frac{1}{2 \pi i} \int_{c-i \infty}^{c+i \infty} \mathrm{d} z \bar{\varphi}(z) x^{-z}
$$


with $c>0$.

Using this representation of $\varphi$ in the definition of $I_{d}$ [see Eq. (A5)] we find

$$
I_{d}(s)=\frac{1}{2 \pi i} \int_{c-i \infty}^{c+i \infty} \mathrm{d} z \bar{\varphi}(z) s^{d / 2-1-z} \frac{\pi}{2 \sin \pi(d / 2-z)},
$$

where $d / 2-1<c<d / 2$. This expression allows us to determine the asymptotic behavior for $s \rightarrow 0$, by shifting the integration contour towards negative values of $\operatorname{Re} z$ :

$I_{d}(s)=\frac{1}{2} \bar{\varphi}(d / 2-1)+\frac{\pi}{2 \sin (\pi d / 2)} \varphi(0) s^{d / 2-1}-\frac{1}{2} \bar{\varphi}(d / 2-2) s+\sum_{k=1}^{\infty}\left[A_{k} s^{d / 2-1+k}+B_{k} s^{1+k}\right]$.

Note that the first term, $I_{d}(0)(d>2)$, depends on the specific choice of the cut-off function $\varphi(x)$, whereas the second term is actually universal provided that $\varphi(0)=1$. Higher-order terms do depend on $\varphi(x)$. Let us consider the linear term is $s$. The analytic extension of $\bar{\varphi}(z)$ to the region $-1<\operatorname{Re}(z)<0$ is readily computed as

$$
\bar{\varphi}(z)=\int_{0}^{\infty} \mathrm{d} x x^{z-1}[\varphi(x)-\varphi(0)]
$$

and therefore

$$
\alpha_{d} \equiv-\frac{1}{2} \bar{\varphi}(d / 2-2)=-\frac{1}{2} \int_{0}^{\infty} \mathrm{d} x x^{d / 2-3}[\varphi(x)-1]
$$

behaves as

$$
\alpha_{4-\epsilon}=\frac{1}{\epsilon}+\text { finite } \text { for } \epsilon \rightarrow 0 .
$$

Accordingly, Eq. (A28) can be cast in the form

$$
I_{d}(s)=I_{d}(0)-\kappa_{d} s^{d / 2-1}+\alpha_{d} s+\sum_{k=1}^{\infty}\left[A_{k} s^{d / 2-1+k}+B_{k} s^{1+k}\right],
$$

where $\kappa_{d}=\pi /[2 \sin (\pi(d-2) / 2)]$, which leads to Eq. (A15). The same conclusion can be drawn by adopting different regularization schemes (see, e.g., Appendix B of Ref. [42]).

\section{APPENDIX B: ONE-LOOP FEYNMAN DIAGRAMS}

As usual in the computations of Feynman diagrams, it is convenient to calculate first the corresponding one-particle irreducible (1PI) parts. For the response functions they are given by the two tadpoles $I_{\sigma}, I_{\boldsymbol{\pi}}$ (see Eqs. (77) and (78)) and by the four "bubbles" which can be written as (hereafter the response and correlation functions appearing in the integrals are the Gaussian ones) 


$$
\begin{aligned}
& B_{L L}(t, s)=\int(\mathrm{d} q) R_{\mathbf{q}}^{\sigma}(t, s) C_{\mathbf{q}}^{\sigma}(t, s)=\frac{2 t^{-3}}{(8 \pi)^{d / 2}} \int_{0}^{s} \mathrm{~d} x x^{3}(t-x)^{-d / 2} \equiv \frac{2}{(8 \pi)^{d / 2}} H_{L}(t, s), \\
& B_{T L}(t, s)=\int(\mathrm{d} q) R_{\mathbf{q}}^{\boldsymbol{\pi}}(t, s) C_{\mathbf{q}}^{\sigma}(t, s)=\frac{t}{s} B_{L L}(t, s)=\frac{2}{(8 \pi)^{d / 2}} \frac{t}{s} H_{L}(t, s), \\
& B_{T T}(t, s)=\int(\mathrm{d} q) R_{\mathbf{q}}^{\boldsymbol{\pi}}(t, s) C_{\mathbf{q}}^{\boldsymbol{\pi}}(t, s)=\frac{2 t^{-1}}{(8 \pi)^{d / 2}} \int_{0}^{s} \mathrm{~d} x x(t-x)^{-d / 2} \equiv \frac{2}{(8 \pi)^{d / 2}} H_{T}(t, s), \\
& B_{L T}(t, s)=\int(\mathrm{d} q) R_{\mathbf{q}}^{\sigma}(t, s) C_{\mathbf{q}}^{\boldsymbol{\pi}}(t, s)=\frac{s}{t} B_{T T}(t, s)=\frac{2}{(8 \pi)^{d / 2}} \frac{s}{t} H_{T}(t, s),
\end{aligned}
$$

where

$$
\begin{aligned}
H_{L}(t, s)= & t^{-3} \int_{0}^{s} \mathrm{~d} x x^{3}(t-x)^{-d / 2} \\
= & A_{L} t^{-1+\epsilon / 2}-A_{L} t^{-1}(t-s)^{\epsilon / 2}+B_{L} t^{-2} s(t-s)^{\epsilon / 2}+C_{L} t^{-3} s^{2}(t-s)^{\epsilon / 2} \\
& +D_{L} t^{-3} s^{3}(t-s)^{-1+\epsilon / 2}, \\
H_{T}(t, s)= & t^{-1} \int_{0}^{s} \mathrm{~d} x x(t-x)^{-d / 2}= \\
= & A_{T} t^{-1+\epsilon / 2}-A_{T} t^{-1}(t-s)^{\epsilon / 2}+B_{T} t^{-1} s(t-s)^{-1+\epsilon / 2},
\end{aligned}
$$

with

$$
\begin{aligned}
& A_{L}=\frac{6}{(2+\epsilon / 2)(1+\epsilon / 2) \epsilon / 2(-1+\epsilon / 2)}, \\
& B_{L}=-\frac{6}{(2+\epsilon / 2)(1+\epsilon / 2)(-1+\epsilon / 2)}, \\
& C_{L}=-\frac{3}{(2+\epsilon / 2)(-1+\epsilon / 2)} \\
& D_{L}=-\frac{1}{(-1+\epsilon / 2)} \\
& A_{T}=\frac{1}{\epsilon / 2(-1+\epsilon / 2)} \\
& B_{T}=-\frac{1}{(-1+\epsilon / 2)} .
\end{aligned}
$$

This parameterization of the 1PI parts of the integrals is particularly convenient, as explained in Ref. [30].

In what follows we shall make extensive use of the results of Ref. [30]. However, we alert the reader to the fact that the factor $r_{d}$ appearing in Ref. [30] has to be identified, in the current notation, with $r_{\sigma}$ and not with $r_{d} \equiv r_{\sigma}+(n-1) r_{\pi} / 3$ (unless differently stated).

\section{Longitudinal response}

The integral $I_{1}$ and $I_{2}$ have been computed in Ref. [30], Eq. (A.9) and (A.11), respectively. $I_{3}$ is easily related to $I_{1}$ :

$$
I_{3}(t, s)=\int_{s}^{t} \mathrm{~d} t^{\prime} R^{\sigma}\left(t, t^{\prime}\right) I_{\boldsymbol{\pi}}\left(t^{\prime}\right) R^{\sigma}\left(t^{\prime}, s\right)=\frac{r_{\boldsymbol{\pi}}}{r_{\sigma}} I_{1}(t, s),
$$


whereas $I_{4}$ is given by

$$
\begin{aligned}
I_{4}(t, s) & =\int_{s}^{t} \mathrm{~d} t_{1} \int_{s}^{t_{1}} \mathrm{~d} s_{1} R^{\sigma}\left(t, t_{1}\right) \sqrt{2} m\left(t_{1}\right) B_{T T}\left(t_{1}, s_{1}\right) \sqrt{2} m\left(s_{1}\right) R^{\sigma}\left(s_{1}, s\right) \\
& =\frac{6}{(8 \pi)^{d / 2}}\left(\frac{s}{t}\right)^{3 / 2} \int_{s}^{t} \mathrm{~d} t_{1} t_{1} \int_{s}^{t_{1}} \mathrm{~d} s_{1} s_{1}^{-2} H_{T}\left(t_{1}, s_{1}\right) .
\end{aligned}
$$

According to the decomposition (B6) of $H_{T}$, this expression can be written as $I_{4}=\sum_{i=1}^{3}\left(a_{i}\right)$, where $\left(a_{i}\right)$ are easily calculable:

$$
\begin{aligned}
\frac{\left(a_{1}\right)}{A_{T}} & =\int_{s}^{t} \mathrm{~d} t_{1} \int_{s}^{t_{1}} \mathrm{~d} s_{1} s_{1}^{-2} t_{1}^{\epsilon / 2}=\frac{t^{1+\epsilon / 2}-s^{1+\epsilon / 2}}{s(1+\epsilon / 2)}-\frac{2}{\epsilon}\left(t^{\epsilon / 2}-s^{\epsilon / 2}\right) \\
-\frac{\left(a_{2}\right)}{A_{T}} & =\int_{s}^{t} \mathrm{~d} t_{1} \int_{s}^{t_{1}} \mathrm{~d} s_{1} s_{1}^{-2}\left(t_{1}-s_{1}\right)^{\epsilon / 2} \\
& =\frac{1}{1+\epsilon / 2}\left[\frac{t}{s}-1-\ln \frac{t}{s}+\frac{\epsilon}{2}\left(\left(\frac{t}{s}-1\right) \ln (t-s)-(1+\ln t) \ln \frac{t}{s}+\frac{\pi^{2}}{6}-\operatorname{Li}_{2}(s / t)\right)\right]+O(\epsilon), \\
\frac{\left(a_{3}\right)}{B_{T}} & =\int_{s}^{t} \mathrm{~d} t_{1} \int_{s}^{t_{1}} \mathrm{~d} s_{1} s_{1}^{-1}\left(t_{1}-s_{1}\right)^{-1+\epsilon / 2}=\frac{2}{\epsilon} \ln \frac{t}{s}+\ln t \ln \frac{t}{s}+\operatorname{Li}_{2}(s / t)-\frac{\pi^{2}}{6}+O(\epsilon) .
\end{aligned}
$$

and whose sum is $(x \equiv s / t)$

$$
I_{4}=\frac{6 s^{\epsilon / 2}}{(8 \pi)^{d / 2}} x^{3 / 2}\left[-\frac{2}{\epsilon} \ln x+\frac{\ln ^{2} x}{2}+\left(\frac{1}{x}-1\right) \ln (1-x)+O(\epsilon)\right] .
$$

\section{Longitudinal correlation}

The integrals $I_{6}$ and $I_{7}$ have been calculated in Ref. [30], Eq (B.25) [see also Eq. (B.26)] and Eq. (B.27), respectively, whereas $I_{10}$ is trivially related to $I_{7}$ :

$$
I_{10}=\frac{r_{\boldsymbol{\pi}}}{r_{\sigma}} I_{7}
$$

$I_{9}$ can be written as

$$
\begin{aligned}
I_{9}(t, s)=\int_{0}^{t} \mathrm{~d} t_{2} \int_{t_{2}}^{t} \mathrm{~d} t_{1} R_{\mathbf{q}=0}^{\sigma}\left(t, t_{1}\right) \sqrt{2} m\left(t_{1}\right) B_{T T}\left(t_{1}, t_{2}\right) \sqrt{2} m\left(t_{2}\right) C_{\mathbf{q}=0}^{\sigma}\left(t_{2}, s\right)+ \\
\quad+\int_{0}^{s} \mathrm{~d} t_{2} \int_{t_{2}}^{s} \mathrm{~d} t_{1} C_{\mathbf{q}=0}^{\sigma}\left(t, t_{1}\right) \sqrt{2} m\left(t_{1}\right) B_{T T}\left(t_{2}, t_{1}\right) \sqrt{2} m\left(t_{2}\right) R_{\mathbf{q}=0}^{\sigma}\left(s, t_{2}\right)
\end{aligned}
$$

which, using Eq. (B3), becomes

$$
\begin{aligned}
I_{9}(t, s)= & \frac{3}{(8 \pi)^{d / 2}}(s t)^{-3 / 2}\left[\int_{0}^{s} \mathrm{~d} t_{2} t_{2}^{2} \int_{t_{2}}^{t} \mathrm{~d} t_{1} t_{1} H_{T}\left(t_{1}, t_{2}\right)\right. \\
& \left.+s^{4} \int_{s}^{t} \mathrm{~d} t_{2} t_{2}^{-2} \int_{t_{2}}^{t} \mathrm{~d} t_{1} t_{1} H_{T}\left(t_{1}, t_{2}\right)+\int_{0}^{s} \mathrm{~d} t_{1} t_{1}^{2} \int_{t_{1}}^{s} \mathrm{~d} t_{2} t_{2} H_{T}\left(t_{2}, t_{1}\right)\right] .
\end{aligned}
$$


We denote the three terms in square brackets by $(A),(B)$, and $(C)$, i.e., $[\ldots]=[(A)+(C)+$ $(C)$ ]. In turn each of them can be written as the sum of the three contributions coming from the decomposition of $H_{T}$ according to Eq. (B6), leading to the following expression for $I_{9}$ :

$$
I_{9}(t, s)=\frac{3(s t)^{-3 / 2}}{(8 \pi)^{d / 2}} \sum_{b \in A, B, C} \sum_{i=1}^{3}(a)_{i} .
$$

The terms with $i=1$ and 2 are the same as the corresponding ones calculated in Ref. [30], Eqs. (B.10), (B.11), (B.15), (B.16), (B.20), and (B.21), with the proviso that $A_{d}$ therein has to be replaced by $A_{T}$ here (see Eq. (B11)). We still need

$$
\begin{aligned}
\frac{(A)_{3}}{B_{T}} & =\int_{0}^{s} \mathrm{~d} t_{2} t_{2}^{3} \int_{t_{2}}^{t} \mathrm{~d} t_{1}\left(t_{1}-t_{2}\right)^{-1+\epsilon / 2} \\
& =\frac{s^{4}}{2 \epsilon}-\frac{s^{4}}{16}-\frac{s^{3} t}{12}-\frac{s^{2} t^{2}}{8}-\frac{s t^{3}}{4}-\frac{1}{4}\left(t^{4}-s^{4}\right) \ln (t-s)+\frac{t^{4}}{4} \log t+O(\epsilon), \\
\frac{(B)_{3}}{B_{T} s^{4}} & =\int_{s}^{t} \mathrm{~d} t_{2} t_{2}^{-1} \int_{t_{2}}^{t} \mathrm{~d} t_{1}\left(t_{1}-t_{2}\right)^{-1+\epsilon / 2}=\frac{2}{\epsilon} \ln \frac{t}{s}+\ln t \ln \frac{t}{s}+\operatorname{Li}_{2}(s / t)-\frac{\pi^{2}}{6}+O(\epsilon), \\
\frac{(C)_{3}}{B_{T}} & =\int_{0}^{s} \mathrm{~d} t_{1} t_{1}^{3} \int_{t_{1}}^{s} \mathrm{~d} t_{2}\left(t_{2}-t_{1}\right)^{-1+\epsilon / 2}=s^{4}\left(\frac{1}{2 \epsilon}-\frac{25}{48}+\frac{1}{4} \ln s\right)+O(\epsilon) .
\end{aligned}
$$

Summing these nine terms we have

$$
I_{9}(t, s)=\frac{3 s^{4+\epsilon / 2}(s t)^{-3 / 2}}{(8 \pi)^{d / 2}}\left[\frac{1-2 \ln x}{\epsilon}+\frac{\ln ^{2} x}{2}-\frac{107}{72}+\mathcal{C}(x)\right],
$$

where

$$
\mathcal{C}(x)=\frac{4}{3} \frac{\ln (1-x)}{x}-\frac{1}{3 x^{4}}\left[\ln (1-x)+x+\frac{x^{2}}{2}+\frac{x^{3}}{3}\right]+\frac{5}{4}-\ln (1-x)
$$

$[\mathcal{C}(0)=0]$

$I_{5}$ has been calculated in Ref. [30], Eqs. (B.5) and (B.6). In order compute $I_{8}$ we first need its 1 PI part [we define $\left.t_{<}=\min \left\{t, t^{\prime}\right\}\right]$

$$
\begin{aligned}
B_{C C}^{T T}\left(t, t^{\prime}\right) & =\int(\mathrm{d} q)\left[C_{\mathbf{q}}^{\boldsymbol{\pi}}\left(t, t^{\prime}\right)\right]^{2}=\int(\mathrm{d} q) 4\left(t t^{\prime}\right)^{-1} \mathrm{e}^{-2 q^{2}\left(t+t^{\prime}\right)} \int_{0}^{t_{<}} \mathrm{d} t_{1} t_{1} \mathrm{e}^{2 q^{2} t_{1}} \int_{0}^{t_{<}} \mathrm{d} t_{2} t_{2} \mathrm{e}^{2 q^{2} t_{2}}= \\
& =\frac{4\left(t t^{\prime}\right)^{-1}}{(8 \pi)^{d / 2}} \int_{0}^{t<} \mathrm{d} t_{1} \int_{0}^{t_{<}} \mathrm{d} t_{2} \frac{t_{1} t_{2}}{\left(t+t^{\prime}-t_{1}-t_{2}\right)^{d / 2}} .
\end{aligned}
$$

so that

$$
\begin{aligned}
I_{8} & =\int_{0}^{s} \mathrm{~d} \tau_{1} \int_{0}^{t} \mathrm{~d} \tau_{2} R^{\sigma}\left(s, \tau_{1}\right) \sqrt{2} m\left(\tau_{1}\right) B_{C C}^{T T}\left(\tau_{1}, \tau_{2}\right) \sqrt{2} m\left(\tau_{2}\right) R^{\sigma}\left(t, \tau_{2}\right) \\
& =3(s t)^{-3 / 2}\left[2 \int_{0}^{s} \mathrm{~d} \tau_{1} \int_{0}^{\tau_{1}} \mathrm{~d} \tau_{2} \tau_{1} \tau_{2} B_{C C}^{T T}\left(\tau_{1}, \tau_{2}\right)+\int_{0}^{s} \mathrm{~d} \tau_{1} \int_{s}^{t} \mathrm{~d} \tau_{2} \tau_{1} \tau_{2} B_{C C}^{T T}\left(\tau_{1}, \tau_{2}\right)\right] \\
& =3(s t)^{-3 / 2}[2 B+A] .
\end{aligned}
$$


Both $A$ and $B$ do not diverge for $d \rightarrow 4$. Therefore no dimensional regularization is required and one can calculate them directly in $d=4$ :

$$
\begin{aligned}
A & =4(8 \pi)^{-d / 2} \int_{0}^{s} \mathrm{~d} \tau_{1} \int_{s}^{t} \mathrm{~d} \tau_{2} \int_{0}^{\tau_{1}} \mathrm{~d} t_{1} \int_{0}^{\tau_{1}} \mathrm{~d} t_{2} \frac{t_{1} t_{2}}{\left(\tau_{1}+\tau_{2}-t_{1}-t_{2}\right)^{2}}+O(\epsilon) \\
& =4(8 \pi)^{-d / 2} s^{4}\left[\mathcal{D}(s / t)+\frac{2}{3} \ln 2-\frac{3}{8}\right]+O(\epsilon), \\
B & =4(8 \pi)^{-d / 2} \int_{0}^{s} \mathrm{~d} \tau_{1} \int_{0}^{\tau_{1}} \mathrm{~d} \tau_{2} \int_{0}^{\tau_{2}} \mathrm{~d} t_{1} \int_{0}^{\tau_{2}} \mathrm{~d} t_{2} \frac{t_{1} t_{2}}{\left(\tau_{1}+\tau_{2}-t_{1}-t_{2}\right)^{2}}+O(\epsilon) \\
& =4(8 \pi)^{-d / 2} s^{4}\left(\frac{7}{24}-\frac{1}{3} \ln 2\right)+O(\epsilon),
\end{aligned}
$$

where

$$
\begin{aligned}
\mathcal{D}(x) & =\frac{1}{8}+\frac{1}{4 x^{3}}+\frac{1}{12 x^{2}}-\frac{1}{12 x}+\ln (1-x)\left(\frac{1}{24}+\frac{5}{24 x^{4}}-\frac{1}{6 x^{3}}-\frac{1}{4 x^{2}}+\frac{1}{6 x}\right) \\
& +\ln (1+x)\left(-\frac{1}{24}-\frac{1}{24 x^{4}}-\frac{1}{6 x^{3}}-\frac{1}{4 x^{2}}-\frac{1}{6 x}\right)
\end{aligned}
$$

$[\mathcal{D}(0)=0]$. Accordingly,

$$
I_{8}=\frac{12}{(8 \pi)^{d / 2}} s\left(\frac{s}{t}\right)^{3 / 2}\left[\frac{5}{24}+\mathcal{D}(x)\right]
$$

Summing all these contributions according to Eq. (92) one finds that the function $f_{C}^{\sigma}$ introduced in Eq. (93) is given by

$$
f_{C}^{\sigma}(x)=\frac{3}{2} \mathcal{A}(x)+\frac{3}{4} \mathcal{B}(x)+\frac{n-1}{12} \mathcal{C}(x)+\frac{n-1}{6} \mathcal{D}(x),
$$

where $\mathcal{A}(x), \mathcal{B}(x)$ are given by Eqs. (B.5) and (B.26), of Ref. [30], whereas $\mathcal{C}(x)$ and $\mathcal{D}(x)$ are given by Eqs. (B24) and (B29) of this appendix.

\section{Transverse response}

The integrals for $I_{11}$ and $I_{12}$ are (see Eq. (A.9) in Ref. [30]):

$$
\begin{aligned}
& I_{11}(t, s)=\int_{s}^{t} \mathrm{~d} t^{\prime} R^{\boldsymbol{\pi}}\left(t, t^{\prime}\right) I_{\sigma}\left(t^{\prime}\right) R^{\boldsymbol{\pi}}\left(t^{\prime}, s\right)=\frac{t}{s} I_{1}(t, s), \\
& I_{12}(t, s)=\int_{s}^{t} \mathrm{~d} t^{\prime} R^{\boldsymbol{\pi}}\left(t, t^{\prime}\right) I_{\boldsymbol{\pi}}\left(t^{\prime}\right) R^{\boldsymbol{\pi}}\left(t^{\prime}, s\right)=\frac{r_{\boldsymbol{\pi}}}{r_{\sigma}} I_{11}(t, s) .
\end{aligned}
$$

$I_{13}$ is, instead, given by

$$
\begin{aligned}
I_{13}(t, s) & =\int_{s}^{t} \mathrm{~d} t_{1} \int_{s}^{t_{1}} \mathrm{~d} s_{1} R^{\boldsymbol{\pi}}\left(t, t_{1}\right) \sqrt{2} m\left(t_{1}\right) B_{T L}\left(t_{1}, s_{1}\right) \sqrt{2} m\left(s_{1}\right) R^{\boldsymbol{\pi}}\left(s_{1}, s\right) \\
& =\frac{6}{(8 \pi)^{d / 2}}\left(\frac{s}{t}\right)^{1 / 2} \int_{s}^{t} \mathrm{~d} t_{1} t_{1} \int_{s}^{t_{1}} \mathrm{~d} s_{1} s_{1}^{-2} H_{L}\left(t_{1}, s_{1}\right),
\end{aligned}
$$


which is just $I_{2}(t, s) t / s$, where $I_{2}$ has been calculated in Eq. (A.11) of Ref. [30]. For $I_{14}$ we have

$$
\begin{aligned}
I_{14}(t, s) & =\int_{s}^{t} \mathrm{~d} t_{1} \int_{s}^{t_{1}} \mathrm{~d} s_{1} R^{\boldsymbol{\pi}}\left(t, t_{1}\right) \sqrt{2} m\left(t_{1}\right) B_{L T}\left(t_{1}, s_{1}\right) \sqrt{2} m\left(s_{1}\right) R^{\pi}\left(s_{1}, s\right) \\
& =\frac{6}{(8 \pi)^{d / 2}}\left(\frac{s}{t}\right)^{1 / 2} \int_{s}^{t} \mathrm{~d} t_{1} t_{1}^{-1} \int_{s}^{t_{1}} \mathrm{~d} s_{1} H_{T}\left(t_{1}, s_{1}\right),
\end{aligned}
$$

which can be split in three parts, according to the parameterization (B6) of $H_{T}$. The final result is

$$
\begin{aligned}
I_{14}(t, s) & =\frac{6 s^{\epsilon / 2}}{(8 \pi)^{d / 2}}\left(\frac{s}{t}\right)^{1 / 2}\left[-\frac{2}{\epsilon} \ln x+\ln x+\frac{\ln ^{2} x}{2}-\frac{\pi^{2}}{3}\right. \\
& \left.+3(1-x)-(1-x) \ln (1-x)+2 \operatorname{Li}_{2}(x)+O(\epsilon)\right] .
\end{aligned}
$$

\section{Transverse correlation}

As usual, the tadpole integrals $I_{15}$ and $I_{16}$ are easy

$$
\begin{aligned}
I_{16}(t, s) & =\int_{0}^{s} \mathrm{~d} t^{\prime} R^{\boldsymbol{\pi}}\left(t^{\prime}, s\right) I_{\sigma}\left(t^{\prime}\right) C^{\boldsymbol{\pi}}\left(t, t^{\prime}\right)+\int_{0}^{t} \mathrm{~d} t^{\prime} R^{\boldsymbol{\pi}}\left(t^{\prime}, t\right) I_{\sigma}\left(t^{\prime}\right) C^{\boldsymbol{\pi}}\left(t^{\prime}, s\right)= \\
& =2 N_{d} r_{\sigma} s\left(\frac{s}{t}\right)^{1 / 2} s^{\epsilon / 2}\left\{\frac{2}{2+\epsilon / 2}+\frac{2}{\epsilon}\left[\left(\frac{t}{s}\right)^{\epsilon / 2}-1\right]\right\}, \\
I_{15}(t, s) & =\frac{r_{\boldsymbol{\pi}}}{r_{\sigma}} I_{16}(t, s) .
\end{aligned}
$$

There are two diagrams involving $B_{L T}\left(t_{1}, s_{1}\right)$ :

$$
\begin{aligned}
I_{17}(t, s) & =\int_{0}^{t} \mathrm{~d} t_{2} \int_{t_{2}}^{t} \mathrm{~d} t_{1} R^{\boldsymbol{\pi}}\left(t, t_{1}\right) \sqrt{2} m\left(t_{1}\right) B_{L T}\left(t_{1}, t_{2}\right) \sqrt{2} m\left(t_{2}\right) C^{\boldsymbol{\pi}}\left(t_{2}, s\right) \\
& +\int_{0}^{s} \mathrm{~d} t_{1} \int_{t_{1}}^{s} \mathrm{~d} t_{2} C^{\boldsymbol{\pi}}\left(t, t_{1}\right) \sqrt{2} m\left(t_{1}\right) B_{L T}\left(t_{2}, t_{1}\right) \sqrt{2} m\left(t_{2}\right) R^{\boldsymbol{\pi}}\left(s, t_{2}\right),
\end{aligned}
$$

which can be written, via Eq. (B4), as

$$
\begin{aligned}
I_{17}(t, s)=\frac{6(s t)^{-1 / 2}}{(8 \pi)^{d / 2}} & {\left[\int_{0}^{s} \mathrm{~d} t_{2} t_{2}^{2} \int_{t_{2}}^{t} \mathrm{~d} t_{1} t_{1}^{-1} H_{T}\left(t_{1}, t_{2}\right)+s^{2} \int_{s}^{t} \mathrm{~d} t_{2} \int_{t_{2}}^{t} \mathrm{~d} t_{1} t_{1}^{-1} H_{T}\left(t_{1}, t_{2}\right)\right.} \\
& \left.+\int_{0}^{s} \mathrm{~d} t_{1} t_{1}^{2} \int_{t_{1}}^{s} \mathrm{~d} t_{2} t_{2}^{-1} H_{T}\left(t_{2}, t_{1}\right)\right] .
\end{aligned}
$$

In turn, we decompose each of the three terms in square brackets in three parts, according to the parameterization (B6) of $H_{T}$. We do not report the details of the calculation, but

only the final result

$$
I_{17}(t, s)=\frac{6 s^{1+\epsilon / 2}}{(8 \pi)^{d / 2}}\left(\frac{s}{t}\right)^{1 / 2}\left[\frac{2}{\epsilon}-\frac{2}{\epsilon} \ln x+\frac{\ln ^{2} x}{2}+\ln x+\frac{23}{18}-\frac{\pi^{2}}{3}+\mathcal{G}(x)+O(\epsilon)\right],
$$


where

$$
\mathcal{G}(x)=-\frac{1}{3}-\frac{20}{9} x-\frac{2}{3} \frac{\ln (1-x)+x}{x^{2}}+\frac{2}{3} x \log (1-x)+2 \operatorname{Li}_{2}(x)
$$

$[\mathcal{G}(0)=0]$.

The expression for $I_{18}$ is

$$
\begin{aligned}
I_{18}(t, s) & =\int_{0}^{t} \mathrm{~d} t_{2} \int_{t_{2}}^{t} \mathrm{~d} t_{1} R^{\boldsymbol{\pi}}\left(t, t_{1}\right) \sqrt{2} m\left(t_{1}\right) B_{T L}\left(t_{1}, t_{2}\right) \sqrt{2} m\left(t_{2}\right) C^{\boldsymbol{\pi}}\left(t_{2}, s\right) \\
& +\int_{0}^{s} \mathrm{~d} t_{1} \int_{t_{1}}^{s} \mathrm{~d} t_{2} C^{\boldsymbol{\pi}}\left(t, t_{1}\right) \sqrt{2} m\left(t_{1}\right) B_{T L}\left(t_{2}, t_{1}\right) \sqrt{2} m\left(t_{2}\right) R^{\boldsymbol{\pi}}\left(s, t_{2}\right),
\end{aligned}
$$

which can be written, using Eq. (B2), as

$$
\begin{aligned}
I_{18}(t, s)=\frac{6(s t)^{-1 / 2}}{(8 \pi)^{d / 2}} & {\left[\int_{0}^{s} \mathrm{~d} t_{2} t_{2}^{2} \int_{t_{2}}^{t} \mathrm{~d} t_{1} t_{1}^{-1} H_{L}\left(t_{1}, t_{2}\right)+s^{2} \int_{s}^{t} \mathrm{~d} t_{2} \int_{t_{2}}^{t} \mathrm{~d} t_{1} t_{1}^{-1} H_{L}\left(t_{1}, t_{2}\right)\right.} \\
& \left.+\int_{0}^{s} \mathrm{~d} t_{1} t_{1}^{2} \int_{t_{1}}^{s} \mathrm{~d} t_{2} t_{2}^{-1} H_{L}\left(t_{2}, t_{1}\right)\right] .
\end{aligned}
$$

We decompose again each of the three terms in square brackets in three parts, according to the parameterization (B5) of $H_{L}$. Also in this case we do not report the details of the calculations, but only the final result

$$
I_{18}(t, s)=\frac{6 s^{1+\epsilon / 2}}{(8 \pi)^{d / 2}}\left(\frac{s}{t}\right)^{1 / 2}\left[\frac{2}{\epsilon}-\frac{2}{\epsilon} \ln x+\frac{\ln ^{2} x}{2}+\frac{3}{2} \ln x+\frac{\pi^{2}}{3}-\frac{17}{3}+\mathcal{F}(x)+O(\epsilon)\right],
$$

where

$$
\mathcal{F}(x)=5+\frac{x}{3}-4 \ln (1-x)-\frac{2}{x^{2}}[\ln (1-x)+x]+6 \frac{\ln (1-x)}{x}-2 \operatorname{Li}_{2}(x)
$$

$[\mathcal{F}(0)=0]$

There is a last $1 \mathrm{PI}$ diagram

$$
\begin{aligned}
B_{C C}^{T L}\left(t, t^{\prime}\right) & =\int(\mathrm{d} q) C_{\mathbf{q}}^{\boldsymbol{\pi}}\left(t, t^{\prime}\right) C_{\mathbf{q}}^{\sigma}\left(t, t^{\prime}\right) \\
& =\int(\mathrm{d} q) 4\left(t t^{\prime}\right)^{-2} \mathrm{e}^{-2 q^{2}\left(t+t^{\prime}\right)} \int_{0}^{t<} \mathrm{d} t_{1} t_{1}^{3} \mathrm{e}^{2 q^{2} t_{1}} \int_{0}^{t_{<}} \mathrm{d} t_{2} t_{2} \mathrm{e}^{2 q^{2} t_{2}} \\
& =\frac{4\left(t t^{\prime}\right)^{-2}}{(8 \pi)^{d / 2}} \int_{0}^{t_{<}} \mathrm{d} t_{1} \int_{0}^{t_{<}} \mathrm{d} t_{2} \frac{t_{1}^{3} t_{2}}{\left(t+t^{\prime}-t_{1}-t_{2}\right)^{d / 2}},
\end{aligned}
$$

in terms of which we express $I_{19}$ as

$$
\begin{aligned}
I_{19} & =\int_{0}^{s} \mathrm{~d} \tau_{1} \int_{0}^{t} \mathrm{~d} \tau_{2} R^{\pi}\left(s, \tau_{1}\right) \sqrt{2} m\left(\tau_{1}\right) B_{C C}^{T L}\left(\tau_{1}, \tau_{2}\right) \sqrt{2} m\left(\tau_{2}\right) R^{\pi}\left(t, \tau_{2}\right) \\
& =3(s t)^{-1 / 2}\left[2 \int_{0}^{s} \mathrm{~d} \tau_{1} \int_{0}^{\tau_{1}} \mathrm{~d} \tau_{2} B_{C C}^{T L}\left(\tau_{1}, \tau_{2}\right)+\int_{0}^{s} \mathrm{~d} \tau_{1} \int_{s}^{t} \mathrm{~d} \tau_{2} B_{C C}^{T L}\left(\tau_{1}, \tau_{2}\right)\right] \\
& =3(s t)^{-3 / 2}[2 B+A] .
\end{aligned}
$$


$A$ and $B$ do not diverge for $d \rightarrow 4$ and therefore one can calculate them directly in $d=4$ :

$$
\begin{aligned}
A & =4(8 \pi)^{-d / 2} \int_{0}^{s} \mathrm{~d} \tau_{1} \int_{s}^{t} \mathrm{~d} \tau_{2} \frac{1}{\left(\tau_{1} \tau_{2}\right)^{2}} \int_{0}^{\tau_{1}} \mathrm{~d} t_{1} \int_{0}^{\tau_{1}} \mathrm{~d} t_{2} \frac{t_{1}^{3} t_{2}}{\left(\tau_{1}+\tau_{2}-t_{1}-t_{2}\right)^{2}}+O(\epsilon) \\
& =4(8 \pi)^{-d / 2} s^{2}\left[\mathcal{E}(s / t)-1-\frac{\pi^{2}}{12}+\frac{8}{3} \ln 2\right]+O(\epsilon), \\
B & =4(8 \pi)^{-d / 2} \int_{0}^{s} \mathrm{~d} \tau_{1} \int_{0}^{\tau_{1}} \mathrm{~d} \tau_{2} \frac{1}{\left(\tau_{1} \tau_{2}\right)^{2}} \int_{0}^{\tau_{2}} \mathrm{~d} t_{1} \int_{0}^{\tau_{2}} \mathrm{~d} t_{2} \frac{t_{1}^{3} t_{2}}{\left(\tau_{1}+\tau_{2}-t_{1}-t_{2}\right)^{2}}+O(\epsilon) \\
& =4(8 \pi)^{-d / 2} s^{2}\left(\frac{29}{48}+\frac{\pi^{2}}{24}-\frac{4}{3} \ln 2\right)+O(\epsilon),
\end{aligned}
$$

with

$$
\mathcal{E}(x)=\frac{1}{2} \int_{0}^{x} \mathrm{~d} y_{1}\left(y_{1}^{2} x^{-2}-1\right) \int_{0}^{1} \mathrm{~d} x_{1} \int_{0}^{1} \mathrm{~d} x_{2} \frac{x_{1}^{3} x_{2}}{\left(1+y_{1}^{-1}-x_{1}-x_{2}\right)^{2}} .
$$

and $\mathcal{E}(0)=0$. Thus

$$
I_{19}=\frac{12}{(8 \pi)^{d / 2}} s\left(\frac{s}{t}\right)^{1 / 2}\left[\frac{5}{24}+\mathcal{E}(x)\right] .
$$

Summing all these contributions according to Eq. (111) (see also Eq. (110)) one finds that the function $f_{C}^{\pi}$ introduced in Eq. (112) is given by

$$
f_{C}^{\pi}(x)=\frac{\mathcal{G}(x)+\mathcal{F}(x)+2 \mathcal{E}(x)}{12},
$$

where $\mathcal{G}(x), \mathcal{F}(x)$, and $\mathcal{E}(x)$ are given by Eqs. (B42), (B46), and (B51), respectively. 


\section{REFERENCES}

[1] L. F. Cugliandolo and J. Kurchan, Analytical Solution of the Off-Equilibrium Dynamics of a Long Range Spin-Glass Model, 1993 Phys. Rev. Lett. 71173 [cond-mat/9303036]; On the Out of Equilibrium Relaxation of the Sherrington - Kirkpatrick model, 1994 J. Phys. A 275749 [cond-mat/9311016]; Weak-ergodicity breaking in mean-field spin-glass models, 1995 Phil. Mag. B 7150 [cond-mat/9403040].

[2] L. F. Cugliandolo, J. Kurchan, and G. Parisi, Off equilibrium dynamics and aging in unfrustrated systems, 1994 J. Phys. I (France) 41641 [cond-mat/9406053].

[3] P. Calabrese and A. Gambassi, Aging in ferromagnetic systems at criticality near four dimensions, 2002 Phys Rev. E 65066120 [cond-mat/0203096]; 2002 Acta Phys. Slov. 52311.

[4] G. Schehr and R. Paul, Universal aging properties at a disordered critical point, 2005 Phys. Rev. E 72016105 [cond-mat/0412447].

[5] E. Vincent, J. Hammann, M. Ocio, J. P. Bouchaud, and L. F. Cugliandolo, Slow dynamics and aging in spin-glasses, 1997 Lect. Notes Phys. 492184 [cond-mat/9607224]; Bouchaud J P, Cugliandolo L F, Kurchan J and Mézard M Out of equilibrium dynamics in spin-glasses and other glassy systems, 1998 in Spin Glasses and Random Fields, Directions in Condensed Matter Physics, vol 12 ed A P Young (Singapore: World Scientific) [cond-mat/9702070];

L. F. Cugliandolo, Dynamics of glassy systems, Lecture notes, Les Houches, July 2002 [cond-mat/0210312];

A. Crisanti and F. Ritort, Violation of the fluctuation-dissipation theorem in glassy systems: basic notions and the numerical evidence, 2003 J. Phys. A 36 R181 [condmat/0212490].

[6] P. Calabrese and A. Gambassi, Aging Properties of Critical Systems: A FieldTheoretical Approach, 2005 J. Phys. A 38 R133 [cond-mat/0410357]

[7] A. Gambassi, Slow dynamics at critical points: the field-theoretical perspective, 2006 J. Phys.: Conf. Series 4013

[8] C. Godrèche and J. M. Luck, Response of non-equilibrium systems at criticality: ferromagnetic models in dimension two and above, 2000 J. Phys. A 339141 [condmat/0001264]; Nonequilibrium critical dynamics of ferromagnetic spin systems, 2002 J. Phys.: Condens. Matter 141589 [cond-mat/0109212]

[9] C. Godrèche and J. M. Luck, Response of non-equilibrium systems at criticality: Exact results for the Glauber-Ising chain, 2000 J. Phys. A 331151 [cond-mat/9911348]

E. Lippiello and M. Zannetti, Fluctuation dissipation ratio in the one dimensional kinetic Ising model, 2000 Phys. Rev. E 613369 [cond-mat/0001103].

[10] P. Calabrese and A. Gambassi, Two-loop Critical Fluctuation-Dissipation Ratio for the Relaxational Dynamics of the $O(N)$ Landau-Ginzburg Hamiltonian, 2002 Phys Rev. E 66066101 [cond-mat/0207452].

[11] P. Calabrese and A. Gambassi, Aging and fluctuation-dissipation ratio for the dilute Ising Model, 2002 Phys. Rev. B 66212407 [cond-mat/0207487].

[12] A. Picone and M. Henkel Response of non-equilibrium systems with long-range initial correlations, 2002 J. Phys. A 355575 [cond-mat/0203411]

[13] Henkel M, Pleimling M, Godrèche C and Luck J M Aging, phase ordering and conformal 
invariance, 2001 Phys. Rev. Lett. 87265701 [hep-th/0107122]

Henkel M, Phenomenology of local scale invariance: from conformal invariance to dynamical scaling 2002 Nucl. Phys. B 641405 [hep-th/0205256]

Henkel M and Pleimling M, Ageing in disordered magnets and local scale-invariance, 2006 cond-mat/0607614

Hinrichsen H, Is local scale invariance a generic property of ageing phenomena? 2006 J. Stat. Mech. L06001 [cond-mat/0603369]

M Henkel, T Enss, and M Pleimling, On the identification of quasiprimary scaling operators in local scale-invariance, 2006 cond-mat/0605211

[14] P. Calabrese and A. Gambassi, Aging at Criticality in Model C Dynamics, 2003 Phys. Rev. E 67036111 [cond-mat/0211062].

[15] P. Mayer, L. Berthier, J. P. Garrahan, and P. Sollich, Fluctuation-dissipation relations in the non-equilibrium critical dynamics of Ising models, 2003 Phys. Rev. E 68016116 [cond-mat/0301493]

P. Mayer and P. Sollich, General Solutions for Multispin Two-Time Correlation and Response Functions in the Glauber-Ising Chain, 2004 J. Phys. A 379 [cond-mat/0307214].

[16] F. Sastre, I. Dornic, and H. Chaté, Nominal Thermodynamic Temperature in Nonequilibrium Kinetic Ising Models, 2003 Phys. Rev. Lett. 91267205 [cond-mat/0308178].

[17] C. Chatelain, A far-from-equilibrium fluctuation-dissipation relation for an IsingGlauber-like model, 2003 J. Phys. A 3610739 [cond-mat/0303545]

F. Ricci-Tersenghi, Measuring the fluctuation-dissipation ratio in glassy systems with no perturbing field, 2003 Phys. Rev. E 68 065104(R) [cond-mat/0307565]

C. Chatelain, On universality in aging ferromagnets, 2004 J. Stat. Mech.: Theor. Exp. P06006 [cond-mat/0404017].

[18] S. Abriet and D. Karevski, Off equilibrium dynamics in 2d-XY system, 2004 Eur. Phys. J. B 3747 [cond-mat/0309342]; Off equilibrium dynamics in the 3d-XY system, 2004 Eur. Phys. J. B 4179 [cond-mat/0405598].

[19] C. Godrèche, F. Krzakala and F. Ricci-Tersenghi, Nonequilibrium critical dynamics of the ferromagnetic Ising model with Kawasaki dynamics, 2004 J. Stat. Mech.: Theor. Exp. P04007 [cond-mat/0401334].

[20] P. Calabrese and A. Gambassi, On the definition of a unique effective temperature for non-equilibrium critical systems, 2004 J. Stat. Mech.: Theor. Exp. P07013 [condmat/0406289]

[21] M. Henkel and G. M. Schütz, On the universality of the fluctuation-dissipation ratio in non-equilibrium critical dynamics, 2004 J. Phys. A 37591 [cond-mat/0308466]

[22] F. Corberi, E. Lippiello, and M. Zannetti, Effective temperature in the quench of coarsening systems to and below $T_{C}, 2004$ J. Stat. Mech.: Theor. Exp. P12007 [condmat/0412088]

E. Lippiello, F. Corberi, and M. Zannetti, Test of Local Scale Invariance from the direct measurement of the response function in the Ising model quenched to and to below $T_{C}$ 2006 cond-mat/0604321

[23] M. Pleimling and A. Gambassi, Corrections to local scale invariance in the nonequilibrium dynamics of critical systems: numerical evidences, 2005 Phys. Rev. B 71 180401(R) [cond-mat/0501483]

[24] A. A. Fedorenko and S. Trimper, Critical aging of a ferromagnetic system from a com- 
pletely ordered state, 2006 Europhys. Lett. 7489 [cond-mat/0507112].

[25] A. Garriga A, P. Sollich P, I. Pagonabarraga, and F. Ritort, Universality of FluctuationDissipation Ratios: The Ferromagnetic Model, 2005 Phys. Rev. E 72056114 [condmat/0508243].

[26] A. Annibale and P. Sollich, Spin, bond and global fluctuation-dissipation relations in the non-equilibrium spherical ferromagnet, 2006 J. Phys. A 392853 [cond-mat/0510731].

[27] Y. Chen and Z.-B. Li, Short-time dynamics of spin systems with long-range correlated quenched impurities, 2005 Phys. Rev. B 71174433

[28] M. Pleimling, Aging phenomena in critical semi-infinite systems, 2004 Phys. Rev. B 70 104401 [cond-mat/0404203]

[29] F. Baumann and M. Pleimling, Out-of-equilibrium properties of the semi-infinite kinetic spherical model, 2006 J. Phys. A 391981 [cond-mat/0509064]

[30] P. Calabrese, A. Gambassi, and F. Krzakala, Critical aging of Ising ferromagnets relaxing from an ordered state, 2006 J. Stat. Mech. P06016 [cond-mat/0604412]

[31] P. C. Hohenberg and B. I. Halperin, Theory of dynamic critical phenomena, 1977 Rev. Mod. Phys. 49435

R. Folk and G. Moser, Critical dynamics: a field-theoretical approach, 2006 J. Phys. A 397943

[32] R. Bausch and H. K. Janssen, Equation of Motion and Nonlinear Response near the Critical point, 1976 Z. Phys. B 25275

[33] R. Bausch, E. Eisenriegler, and H. K. Janssen, Nonlinear Critical Slowing Down of the One-Component Ginzburg-Landau Field, 1979 Z. Phys. B 36179

[34] J. Zinn-Justin, Quantum Field Theory and Critical Phenomena, 3rd edition (Clarendon, Oxford, 1996).

[35] H. K. Janssen, On a Lagrangian for classical field dynamics and renormalization group calculations of dynamical critical properties, 1976 Z. Phys. B 23 377;

R. Bausch, H. K. Janssen, and H. Wagner, Renormalized field theory of critical dynamics, 1976 Z. Phys. B 24 113;

C. De Dominicis, A Lagrangian version of Halperin-Hohenberg-Ma models for the dynamics of critical phenomena, 1975 Nuovo Cim. Lett. 12 567;

C. De Dominicis, Techniques de renormalisation de la théorie des champs et dynamique des phénomène critiques, 1976 J. Physique Coll. 37 C1-247;

C. De Dominicis and L. Peliti, Field-theory renormalisation and critical dynamics above $T_{c}$ : helium, antiferromagnets, and liquid-gas systems, 1978 Phys. Rev. B 18353.

[36] H. K. Janssen, B. Schaub, and B. Schmittmann, New universal short-time scaling behaviour of critical relaxation processes, 1989 Z. Phys B 73539.

[37] H. K. Janssen, On the renormalized field theory of nonlinear critical relaxation, in From Phase Transitions to Chaos- Topics in Modern Statistical Physics, edited by G. Györgyi, I. Kondor, L. Sasvári, T. Tel (World Scientific, Singapore, 1992).

[38] L. F. Cugliandolo, J. Kurchan, and L. Peliti, Energy flow, partial equilibration and effective temperatures in systems with slow dynamics, 1997 Phys. Rev. E 553898 [cond-mat/9611044].

[39] Chamon C, Cugliandolo L. F., Yoshino H., Fluctuations in the coarsening dynamics of the $\mathrm{O}(\mathrm{N})$ model: are they similar to those in glassy systems? 2006 J. Stat. Mech. P01006 [cond-mat/0506297] 
[40] Pelissetto A and Vicari E 2002 Phys. Rep. 368549 [cond-mat/0012164]

[41] H. E. Stanley, Spherical Model as the Limit of Infinite Spin Dimensionality, 1968 Phys. Rev. 176718.

[42] M. Moshe and J. Zinn-Justin, Quantum Field Theory in the Large N Limit: a review, 2003 Phys. Rep. 38569 [hep-th/0306133] 